Title page:

Phase I/II parallel double-blind randomized controlled clinical trial of perispinal etanercept for chronic stroke: improved mobility and pain alleviation.

Word count: 13,171 


\title{
Phase I/II parallel double-blind randomized controlled clinical trial of perispinal etanercept for chronic stroke: improved mobility and pain alleviation.
}

\begin{abstract}
:
Background: Previous open-label studies showed that chronic post-stroke pain could be abated by treatment with perispinal etanercept, although these benefits were questioned. A randomized double-blind placebo controlled clinical trial was conducted to test perispinal etanercept for chronic post-stroke pain.
\end{abstract}

Research design and methods: Participants received two treatments, either with perispinal etanercept (active) or saline (control). Primary outcomes were the differences in mean daily pain levels between groups analysed by SPSS.

Results: On the 0-100 point visual analogue scale, perispinal etanercept reduced mean levels for worst and average daily pain from baseline after two treatments by $19 \cdot 5-24 \%(\mathrm{p}<0 \cdot 05)$, and pain alleviation was maintained in the etanercept group, with no significant change in the control group. $30 \%$ of etanercept participants had near complete pain abatement after first treatment. Goniometry of the paretic arm showed improved mean shoulder rotation by 55 degrees in active forward flexion for the etanercept group $(\mathrm{p}=0 \cdot 003)$, but no change in the control group.

Conclusions: Perispinal etanercept can provide significant and on-going benefits for the chronic post-stroke management of pain and greater shoulder flexion by the paretic arm. Treatment effects are rapid and highly significant, supporting direct action on brain function. 


\section{Introduction:}

\section{- Scientific background, clinical relevance, rationale and objectives}

Major impairment after stroke is exacerbated when accompanied by chronic, severely debilitating and intractable long-term pain $[1,2]$. Strokes in different regions of the brain are often associated with the occurrence of central post-stroke pain (CPSP). CPSP is a neuropathic pain disorder, arising from a combination of both central [3] and peripheral nervous system mechanisms [4]. CPSP is a highly intractable disorder with significant health burdens [1, 2], as is stroke disability itself. A recent systematic review of clinical trials for CPSP has shown no beneficial effects for any other experimental treatments [5]. CPSP frequently requires the use of strong analgesics which may result in further significant impairment and reduced quality of daily life. Often, patients also demonstrate clinical features such as depression and have greater risk of suicide [2]. Hence, finding a beneficial therapy is imperative.

The common proinflammatory cytokine, tumor necrosis factor (TNF) is involved in all phases of stroke, including rehabilitation (reviewed in [6]). Initially, during stroke, TNF is synthesized and released by astrocytes, microglia and neurons in response to ischemia and is a major factor in the pathophysiological processes of stroke. TNF activates the microglia and astrocytes, affects the blood brain barrier permeability, and can adversely affect synaptic transmission and synaptic plasticity during stroke, including rehabilitation [7].Tumor necrosis factor (TNF) has long been implicated as a key factor in the post-stroke neuroinflammatory response with greater levels found in the CSF and plasma $[8,9,10]$ correlating with severity of symptoms as a mediator of focal ischemic brain injury [11]. Elevated TNF levels not only exist in the cerebral spinal fluid of acute stage $[9,12,13,14,15,16,17,18]$ but also chronic post-stroke patients [19] with increased TNF 
expression found elevated in post-mortem brain persisting long after the initial stroke episode [20]. TNF has been established as an integral mediator of neuropathic pain $[21,22,23]$.

For over two decades, research studies have shown the relief of neuropathic pain, recognised and reported for chronic stroke with the use of the TNF blocker, etanercept - an agent comprising immunoglobulin fused with the soluble TNF receptor domain (reviewed in $[24,25,26]$ ). Studies with tracers also showed that administering large molecules such as etanercept via injection into the perispinal space facilitates their uptake into the cerebrospinal venous plexus thereby providing an effective method for direct delivery into the brain through the choroid plexus [27, 28, 29]. Such treatments were also shown to alleviate neuropathic pain in rat models [27, 29] and in human stroke patients [30, 31]. Previous observational studies reported that perispinal etanercept (PSE) therapy provided rapid improvements (within 30-60 minutes after treatment) in stroke related disabilities, including pain [31]. It was concluded that perispinal delivery of etanercept was mediating the rapid actions by a direct effect of blocking TNF in the central nervous system [27, $28,29,30,31,32]$. These reports were met with controversy such that the American Academy of Neurology published a practice advisory in 2016 noting that the evidence to support or refute a benefit of etanercept for treatment of post-stroke disability was insufficient to determine the treatment's effectiveness [33]. This trial was designed to resolve whether the perispinal etanercept injection procedure developed and used in the clinic by Dr E. Tobinick to treat post stroke patients for pain and other dysfunction was in fact successful and worthwhile. Furthermore, the methods followed were exactly those approved and used by Dr Tobinick and have been well documented $[31,34,35,36,37,38,39,40,41]$. Therefore, we undertook the first randomized double-blind clinical trial to determine the effects of two treatments at day 1 and day 14 of perispinal etanercept therapy with primary outcome measures examining the differences from baseline levels in patients with constant daily post-stroke pain to day 30 , two weeks after the second treatment on trial. 
Keywords: clinical trial, perispinal etanercept, reduced post-stroke pain, increased flexion.

\section{Patients and methods:}

The CONSORT guidelines were followed in the preparation of this publication.

\section{Description of trial design including allocation ratio.}

This was a double-blind randomized controlled parallel trial with 1:1 allocation ratio between individuals receiving either etanercept (active) treatment or saline (placebo) control. Approval of the study was obtained from the Griffith University Human Research Ethics committee (MSC/10/14/HREC). Subject applications were clinically evaluated by a neurologist during the screening process for eligibility. The study protocol was fully explained to participants. Informed and written consent was obtained before participation with allocation based on the numerical value assigned upon enrolment into either the etanercept or control group (Fig 1). Trial clinical investigators including the neurologist were involved in the enrolment of participants.

\section{Insert Figure 1 here.}

Specific inclusion criteria used for screening were initially based on the following four requirements:

- $\quad$ aged between $30-80$ years old

- $\quad$ stroke occurring at least 6 months and not more than 15 years prior to screening for this study

- chronic neurological impairment, including hemiparesis, following an ischemic stroke in the territory of the middle cerebral arteries (MCA) (including MCA clot or embolus or carotid occlusion causing MCA territory stroke) or basal ganglia form of intracerebral haemorrhage. 
- constant daily pain post-stroke incorporating one or both ipsilateral limbs and experiencing intractable chronic post-stroke pain with hemiplegic post-stroke shoulder pain and central poststroke pain. The post-stroke pain is of moderate-to-severe in intensity, with a daily average intensity between $4-8$ inclusive on an 11-point (0-10) vertical Numerical Pain Rating Scale supplemented with a faces pain scale (vNPRS-FPS) [42].

- The post-stroke pain is in an area of the body affected by the stroke.

As aphasia is recognized as less common in patients with right than left sided MCA strokes, this randomized, parallel group controlled clinical trial was initially aimed at studying the clinical effects of perispinal delivery of etanercept versus saline in a cohort of participants with chronic ischemic stroke in the territory of the right MCA. In addition to pain as a primary outcome measure, shoulder flexion, spasticity, cognition, executive function, hemispatial neglect and poststroke depression were examined, some as exploratory outcome measures because it was unknown whether the trial would be adequately powered for these particular endpoints.

Before participating, all participants underwent physical examination and vital signs were recorded. All patients were informed prior to trial that any previous medication used regularly was to be maintained and not altered during the period from two weeks before visit 1 on trial until day 30 after visit 1.

\section{Important changes to methods after trial commencement (such as eligibility criteria), with}

reasons: The initial cohort was based on right MCA stroke but due to the limited numbers of available patients presenting that met the initial criteria, enrolment was widened to expand the age limit to 27 and to include non-aphasic stroke patients with left MCA strokes and right-sided impairment or basal ganglia strokes. Applicants were reviewed on a case by case basis by the neurologist and assessed for their capacity to understand and communicate during verbal testing. 


\section{Eligibility criteria for participants:}

Inclusion criteria: At the time of recruitment, all participants were required to travel to the study site and speak fluent English to facilitate effective communication regarding their pain levels. Participants started a pain diary including listing all medications taken from day -7 prior to visit 1 and were required to score their vNPRS-FPS through to completion at day 30 after visit 1 , which were collected. For the complete list of specific inclusion/exclusion criteria, including neurological details of stroke diagnosis, refer to Supplementary Table 1.

\section{Settings and locations where the data were collected}

Patient medical histories relating to their stroke and hospital discharge summaries were collected via email or as hard copies for evaluation by the study investigators during enrolment. All data was collected, bound and stored with Case Report Forms for each participating subject at the Clinical Trials Unit, G40 Health Centre, Griffith University Gold Coast campus, Southport Queensland.

Interventions and Administering procedure: Two active or control treatments (the first at visit 1/day 1 and the second at visit 2/day 14) on trial were administered with all injections doubleblinded to the principal medical investigators and participants. Assessments measuring the responses of participants to treatments were also undertaken in a blinded manner. Treatments were to be halted in the event of an adverse reaction or participant request to discontinue participation. Each etanercept (ENBREL ${ }^{\circledR}$, Pfizer, USA) single-use injectable dose was prepared by solubilizing with the addition of $1.8 \mathrm{cc}$ of non-bacteriostatic sterile water into a $25 \mathrm{mg}$ lyophilized powdered vial of etanercept (containing $10 \mathrm{mg} / \mathrm{mL}$ sucrose, $5 \cdot 8 \mathrm{mg} / \mathrm{mL}$ sodium chloride, $5 \cdot 3 \mathrm{mg} / \mathrm{mL}$ L-arginine hydrochloride, $2 \cdot 6 \mathrm{mg} / \mathrm{mL}$ sodium phosphate, monobasic, monohydrate, and $0.9 \mathrm{mg} / \mathrm{mL}$ sodium phosphate, dibasic, anhydrous). A single dose was 
administered by injection overlying the spine, as described previously $[31,34,35,36,37,38,39$, $40,41]$. Thus, each dose was delivered to the participant in a sitting position with their head bent forward, reaching the chin towards the chest and with the neck horizontal. The injection was given subcutaneously into the posterior cervical interspinous midline (into the interspace midway between C6-C7 or C7-T1 vertebra) using a 27 gauge, half inch needle, free-hand guided and inserted fully into the skin at an angle near perpendicular to the surface. The injection was quickly followed by Trendelenburg positioning, with participant supine on an inversion table and the head dependent for four minutes at an incline of 45 degrees. This approach is in order to effect entry into the cerebrospinal venous system (CSVS) as a previously validated delivery to the brain via the choroid plexus $[27,28,29]$.

Control: The control treatment was sterile saline (suitable for human injection) as a clear colourless solution, with the same appearance as for the etanercept, and prepared in the same type of syringe with the same volume of $1.8 \mathrm{ml}$ as for the test drug. The control was administered using the identical perispinal injection procedure as for the active drug treatment described above.

Outcome Measures: Measures are underlined below.

Primary Outcome Measures: PAIN. On the vertical Numerical Pain Rating Scale (vNPRS), patients were asked where they would mark the number between 0 and 10, or 0 and 100 that fits best to their pain intensity. Zero represented 'no pain at all' whereas the upper limit represented 'the worst pain imaginable'. The 11 point vertical Numeric Pain Rating Scale supplemented with a Faces Pain Scale (vNPRS-FPS) [42] was provided to patients in the weeks preceding trial visit 1. This was used to assess their levels of pain and familiarize prospective trial participants with the pain test as a guide for self-reporting daily pain intensities recorded on their pain diary from day -7 before participation on trial, until day 30 . Participant pain levels were also assessed by 
interview using an expanded $0-100$ point $(1 \mathrm{~cm} / 5$ point numeric interval on the vertical visual analog scale with 2 emoticon face indicators: one for 'no pain' at 0 and one for 'worst pain imaginable' at 100) vNPRS-FPS placed before patients during trial visits 1 and 2, recording both pre- and post-treatment scores, as well as recorded on day 30 by phone interview. Average and Worst levels of Pain: Changes in mean vNPRS-FPS (\% change in pain intensities on the 0-100 point scale) for values recorded from visit 1 before treatment (the baseline (PRE) values) compared with on the final day of participation on trial (day 30 after visit 1; D30). Participants were asked at visit 1 and 2 on trial before and after treatment to rate their "average" and "worst" pain levels considering the last 8 hours and the previous week.

Secondary Outcome Measures: Average level of Pain: Change in mean vNPRS-FPS scores (0100 point scale) from baseline compared with day 1 (visit 1 after treatment) and day 14 (visit 2 after treatment), where the subject was rated for his or her "average pain" over the last 8 hours and considering the previous week. Worst level of Pain: Change in mean vNPRS-FPS score (0100 point scale) from baseline compared with visit 1 and 2 after treatment, where the subject was rated for his or her "worst pain" over the last 8 hours and considering the previous week. Instant Change in Worst Level of Pain: between 30-60 minutes before treatment on day 1 (visit 1) to 3060 minutes after treatment at visit 1 . Shoulder Flexion/Spasticity: mean change in paretic arm by angle of shoulder flexion (active and passive) (measured in degrees of rotation by goniometry) before and after treatment at each visit on day 1 and day 14. Cognition: mean change in Montreal Cognitive Assessment (MOCA)[43] score from baseline on day 1 compared to day 14 (visit 2, after treatment). The Albert's Line Bisection Test is used to detect unilateral visuospatial neglect [44]. Hemispatial Neglect: mean change in Albert's Line Bisection Test from baseline on day 1 to day 14 (visit 2, after treatment). Fatigue Assessment Scale (FAS): mean change in FAS level (out of 50) from baseline to day 30. The FAS has 10-items as statements about different aspects 
of fatigue, each rated from 1 to 5 (1, never; 2, sometimes; 3, regularly; 4, often; and 5, always) and is a valid and reliable test in stroke with higher scores indicating greater fatigue [45].

Exploratory: The Clock Drawing Test (CDT) is used as a psychometric measure for mild to moderate cognitive impairment [46]. Cognition: mean change in CDT score from day 1 of treatment to day 14 (visit 2, after treatment). Motor Function/Balance: mean change in time to complete the five times Sit-To-Stand test from day 1 to day 14 (visit 2, after treatment). Psychological/behavioral function: mean change in Beck's Depression Inventory (BDI) scores from day 1 to day 14 (visit 2, after treatment). Hemispatial Neglect (Instant change, in participants with hemispatial neglect on day 1): mean change in Albert's Line Bisection Test score from 30-60 minutes before treatment on day 1 to 30-60 minutes after treatment on day 1. Thermosensory analysis: mean change in pain detection and pain thresholds using the TSA II thermosensory device (Medoc Advanced Medical Systems) from day 1 (visit 1, prior to treatment) to day 14 (visit 2 after treatment).

Changes to trial outcomes after the trial commenced, with reasons. A Fatigue Assessment Scale (FAS) test, shoulder flexion as well as algometry (ALG) were added as secondary outcome measures to assess changes in patient fatigue levels and sensitivity to pressure. A Force 10 FDX25 force gauge (Wagner Instruments) in peak mode was used for algometry over the medial area of the lower anterior arm regions, repeated three times on each arm and averaged over triplicate measures in Newtons for analysis. The Medoc TSA II Quantitative Sensory Thermoanalyser was also included to evaluate the mean of triplicate tests for patient thermal detection and pain sensitivity over the medial forearm $[47,48]$.

Sample Size and Power Estimation: The sample size of 20 patients on trial with 10 completed in each study group (at least 10 control and 10 etanercept participants) was based on the published 
data from observational studies reported previously $[31,35]$. The assumption was that the population of participants and their outcomes were normally distributed. Dr David Schoenfeld's Harvard website http://hedwig.mgh.harvard.edu/sample_size/js/js_parallel_quant.html was used for sample size determination. This was validated by the trial biostatistician. For the outcomes used in this study, power estimations were as follows:

Power calculation for sample size based on previous reports of analyses by pain test. The values used for this power calculation were taken from those reported [31] (Table 10), obtained after one injection of perispinal etanercept treatment. From this study, the group baseline for the vertical assessment scale (VAS) mean score (_S.D.) was $7 \cdot 1( \pm 2 \cdot 09)$ on the 11 point scale [31]. After treatment, the mean score $( \pm$ SD) was $2 \cdot 3( \pm 2 \cdot 81)$. Based on this data, a minimum total of 14 patients ( 7 in each group) were required for enrolment with power to detect a treatment difference $=83 \%$ at a two-sided 0.05 significance level. Hence, given the predicted size effect, the study was considered suitably powered with a minimum number of patients at 20 to reach levels of significance.

Interim analyses and stopping guideline: After the first randomized group reached 10 patients on both the control and etanercept groups (total of $n=26$ enrolled patients with $n=22$ participants completing week 4/day 30 on trial), interim unblinding and analysis of outcomes was triggered.

Method used to generate the random allocation sequence: A computer based random number generator in blocks of five was used by the pharmacist to establish the trial unblinding code for random assignment of enrolled patients into either group (allowing up to a total of at least 40 patients in each group, if required).

Randomization, Blinding and Patient Replacement Procedures: Patients were assigned based on the randomization code if they met all the inclusion criteria and none of the exclusion criteria. 
Every subject who passed initial screening based on inclusion/exclusion testing was included as intent to treat as they were assessed and entered numerically into the test pool. A final round of screening occurred as patients attended the first treatment, visit 1 at the clinic for validation as suitable for enrolment. A numbered ID was assigned to each participant by the trial pharmacist. Any patient on trial who discontinued or failed to complete the study was replaced and the replacing patient denoted with the next numbered ID in the order of the randomization number code. Allocation was concealed from clinical investigators, assessors and participants during the trial to ensure double-blinding.

Interventions were prepared by the pharmacist to be identical in appearance and placed inside containers labelled only with patient ID and number on enrolment, with the identification of the intervention sealed inside an unblinding envelope at the bottom of the containers and to be opened only in the event of emergency.

\section{Statistical methods used to compare groups for primary and secondary outcomes:}

For statistical analyses, Statistical Package for Social Sciences (SPSS; Vn25) software program was used, available at Griffith University. The data from all analyses of each individual participant were recorded on their Case Report Form and data from this entered onto spreadsheets using Microsoft Excel and then processed via SPSS for generalized estimating equations with first-order autoregressive relationship as the working correlation matrix. Other analyses included two-tailed paired or independent-samples t-tests, ANCOVA, logistic regression using the general linear model with repeated measures or non-parametric and other suitable tests as required. Univariate analysis of variances (UNIANOVA) was used to provide an estimation of effect size $\left(\eta^{2}\right.$; eta squared value) and observed power. Analyses included comparison of outcomes within and between the etanercept and the control groups for mean scores from cognitive function, 
sensory, motorneuron or other tests, as well as differences from baseline. The data analyses included changes from baseline scores with standard error about the mean or interquartile ranges about the changes in median levels. Assumptions of normality were satisfied using ShapiroWilks test, normal Q-Q plots of differences and box-plot outlier analysis. For Mann-Whitney U exact tests, the treatment effects on pain levels (difference between groups) were quantified using the Hodges-Lehmann (HL) estimator from SPSS. This estimator (HL $\Delta$ ) was used to determine the median of all possible differences in outcomes between each subject in the etanercept group versus each subject in the control group. A non-parametric 95\% confidence interval for HL $\Delta$ accompanied these estimates and determined the median of differences between the two groups or the location shift in the median. For analysis of thermal perception and pain thresholds, multilevel modeling with the linear mixed models (LMM) function in SPSS was also used. Bonferroni corrections were applied for multiple comparisons. Where relevant for all the above analyses, $p$-values $<0.05$ were considered significant and $<0.01$ highly significant.

Four participants initially enrolled into the trial were excluded. From the etanercept group, one developed shingles after visit 1 , one had complications of severe lower back pain from spinal stenosis considered unrelated to stroke, and one demonstrated delusional features and hence, was unreliable for assessment. One patient was excluded from the control group after starting oxycodone medication the week of visit 1 and on Day 1, no longer experienced any pain. Patients were recruited over the period from November 2016 through to March 2019.

Decision for study completion: After interim review of the first cohort ( $\mathrm{n}=22$ participants) showed significance $(p<0 \cdot 05)$ across both primary (baseline to day 30 change in $\%$ vNPRS-FPS pain measures on 0-100 scale) and secondary outcomes (pre-post visit 1 change in vNPRS-FPS and shoulder flexion in arc degrees by goniometry), the completion phase was triggered. The study was then stopped early due to the significance of the positive results. Of the completing 
participants, for each individual, all four data points (baseline; visit 1 after treatment 1; visit 2 after treatment 2; and day 30) were included in the vNPRS analysis and by original assigned groups with $\mathrm{n}=10$ in the active and $\mathrm{n}=12$ in the control group used for comparison.

\begin{abstract}
All important harms or unintended effects in each group: No serious adverse events were recorded on trial. However, a single adverse event occurred with one patient developing shingles after visit treatment 1 , a recognized risk previously noted with anti-TNF medication $[49,50]$. Consequently, this patient was excluded from further participating on-trial as the results would be complicated by the pain from shingles and the possibility of post-herpetic neuralgia.
\end{abstract}




\section{Results:}

Of the 26 CPSP patients enrolled on the trial, 22 completed the protocol. No serious adverse events (SAEs) from treatments occurred in the completing participants, with only one adverse event (AE) due to a case of shingles. The distribution of the study group and participant baseline characteristics are shown in Table 1. All participants initially demonstrated significant intractable and constant daily CPSP with pain scores at baseline entry between $40-80$ inclusive on the 0 - 100 point vNPRS-FPS, with their pain refractory to analgesic medications (including oxycodone or pregabalin). Seven participants in the etanercept and five in the control group had limited shoulder flexion of their paretic arm with active motion $\leq 75$ degrees at baseline. No significant baseline differences between the groups were noted for any of the trial measures by independent samples t-test ( $p>0.05$; Table 1) and no unequal variances by Welch's t-test.

Insert Table 1 here.

\section{Primary Outcome Measures:}

\section{Significant decline in pain severity of patients receiving perispinal etanercept compared to} control.

The change from baseline in $\%$ pain intensities (0-100 point scale on the vNPRS-FPS) for the mean values over the four repeated measures of average pain (including baseline (PRE); visit 1 (V1) after treatment 1; visit 2 (V2) after treatment 2; and day 30 (D30) scores) for each group were compared (Fig. 2A).

Insert Figure 2 here.

Analysis by original assigned group of the repeated measures using generalized estimating equations showed a significant group*treatment interaction for worst pain (Wald $\chi^{2}=4 \cdot 58$; df $1 ; p$ 
$=0.032$ ) and for average pain (Wald $\chi^{2}=4 \cdot 161$; df $1 ; p=0 \cdot 041$ ). Post-hoc analysis demonstrated a greater reduction in pain levels for the etanercept compared to the control group over the trial period with a mean decline ( $\%$ change on $0-100$ point scale \pm S.E. $)$ in average and worst daily pain levels from baseline to day 30 within the etanercept group of $24 \pm 9 \%$ and $19 \cdot 5 \pm 6 \cdot 6 \%$, respectively (Fig. 2A,B). The decline in pain levels from baseline to day 30 within the etanercept group for both the average and worst daily pain levels were significant (two-tailed paired t-test: $\mathrm{t}_{(9)}=2 \cdot 63 ; p=0 \cdot 027 ; \mathrm{t}_{(9)}=2 \cdot 94 ; p=0 \cdot 017$, respectively).

Mann Whitney $U$ test showed a significant reduction in both average and worst pain scores comparing the $\%$ differences in vNPRS-FPS (0-100 point scale) from baseline to day 30 after the two perispinal etanercept treatments (Fig. 2B; for average pain: $\mathrm{U}=29 \cdot 5 ; p=0 \cdot 04$, HL $\Delta$ between group medians $=15 ; 95 \% \mathrm{CI}=0-40$; for worst pain: $\mathrm{U}=26 ; p=0 \cdot 023, \mathrm{HL} \Delta$ between group medians $=15 ; 95 \% \mathrm{CI}=5-30)$. By UNIANOVA, the $\%$ differences comparing between groups for changes in mean vNPRS-FPS scores from baseline to day 30 after treatment were also significant (for worst pain: $p=0 \cdot 037, \eta^{2}=0 \cdot 20$, observed power of $56 \%$ and for average pain, $p$ $=0 \cdot 045, \eta^{2}=0 \cdot 19$, observed power of 53\%). At the individual level, 4 of the 10 patients in the etanercept group showed no or limited effects on pain, whereas 3 others had rapid and complete or almost complete resolution of their pain levels directly (by 30 minutes) post-treatment during visit 1.

Within the control group, there was no significant change in the mean values for the average or worst daily pain levels (0-100 point scale) comparing baseline to day 30 (paired t-tests: average pain, $\mathrm{t}_{(11)}=0.52 ; p>0.5$ and worst pain, $\left.\mathrm{t}_{(11)}=0.59 ; \mathrm{p}>0.5\right)$. Categorically, one participant in the control group demonstrated a response for average pain during the trial (Fig. 2B; outlier). 


\section{Secondary Outcome Measures:}

\section{1) Instant differences in worst and average pain.}

For the secondary outcome measures, within the etanercept group a significant difference was detected in the immediate effects from baseline at visit 1 compared to directly after treatment on the $0-100$ point scale (by 30 minutes) for the $\%$ change in worst and average pain levels (paired ttest: $\mathrm{t}_{(9)}=3 \cdot 24 ; p=0 \cdot 01$ and $\mathrm{t}=2 \cdot 59_{(9) ;} \mathrm{p}=0 \cdot 029$, respectively). Post-hoc tests demonstrated that for this instant pain reduction, the mean values ( $\%$ change on the $0-100$ point scale \pm S.E. $)$ for worst pain decreased by $33 \cdot 5 \pm 10 \cdot 4 \%$ within the etanercept group. By comparison, a $10 \cdot 33 \pm 6 \cdot 7$ $\%$ decrease was detected within the control group for the change in worst pain after first treatment, which was non-significant (paired $\mathrm{t}$ test: $\mathrm{t}_{(11)}=1.98 ; p>0.05$ ). Pain scores in the control group also returned to baseline levels by day 30 , indicating no effects of the saline control on pain.

\section{2) Improvements in functional mobility.}

At baseline, the limited extent of shoulder flexion by the paretic arm by all participants (comparing arc degrees of ROM across the entire cohort) was highly correlated comparing the active and passive movements (Pearson's correlation: $\mathrm{r}=0 \cdot 898 ; p<0 \cdot 01$ ). Analysis of repeated measures using generalized estimating equations for changes in mean active shoulder flexion range of motion (ROM/ degrees of arc) by the paretic arm demonstrated a highly significant group*treatment interaction (Wald $\chi^{2}=8 \cdot 625$, df $1 ; p=0 \cdot 003$; Fig. 3A).

Insert Figure 3 here.

Post-hoc analysis showed successive improvements occurred within the etanercept treated group for their active ROM when compared to baseline, initially shoulder flexion improving by a change in mean $( \pm$ S.E. $)$ of $30 \pm 7 \cdot 3$ arc degrees after treatment 1 within $30-60$ minutes (paired t-test: $t_{(9)}$ $=4.07 ; \mathrm{p}=0.003)$, which increased to $55 \pm 12$ arc degrees after treatment 2 (paired $\mathrm{t}$-test: $\mathrm{t}_{(9)}=$ 
$4 \cdot 54 ; p=0.001)($ Fig. 3A). UNIANOVA demonstrated a significant difference between groups in arc degrees of shoulder flexion from baseline to after treatment at visit $2\left(p=0 \cdot 011, \eta^{2}=0 \cdot 28\right.$, observed power of $76 \%$ ). Categorically, 9 out of the 10 patients from the etanercept group with restricted paretic arm mobility had improved shoulder flexion of their paretic arm, such that 6 of these 10 showed marked improvements in active shoulder flexion by an increase $\geq 60$ arc degrees, 3 of the 10 fully regaining 180 degrees of flexion. Again, as with the changes seen with the reduced pain levels, this improvement was noted to begin immediately (by 30 minutes) after the first treatment during visit 1 (Fig. 3A).

A significant group*treatment interaction was also demonstrated for changes in mean passive flexion ROM for the paretic arm (Fig. 3B; Wald $\chi^{2}=4 \cdot 861$, df $1 ; p=0 \cdot 027$ ). Changes from baseline comparing the active to passive flexion ROM within the etanercept treated group were strongly correlated (Pearson's correlation, $\mathrm{r}=0 \cdot 805 ; p<0 \cdot 01$ ), indicating that the etanercept effect was improving both aspects of mobility for the paretic arm. No significant effect on either active or passive shoulder flexion by the paretic arm was detected within the 9 out of 10 in the control group with restricted mobility (Fig. 3; paired t-test, $\mathrm{t}_{(8)}=0.828 ; \mathrm{p}>0.5$ for active; $\mathrm{t}_{(8)}=-1.076 ; \mathrm{p}=$ 0.313 for passive). It should be noted that one of the patients in the control group (the outlier in Fig. 1B), regained complete 180 degree flexion during the trial.

No significant relationship was detected when comparing the decreased pain levels and increased shoulder flexion of the paretic arm in the etanercept group $(\mathrm{r}=0 \cdot 001, \mathrm{p}>0 \cdot 05)$. Categorically, although the majority of patients in the etanercept group showed improvements in shoulder flexion for their paretic arm, one patient in this group had significant pain reduction, but without any accompanying changes in arm mobility, whereas another four from this group, whilst showing limited changes in their pain levels, had greatly improved shoulder flexion. 


\section{Pressure pain sensitivity:}

Analysis by algometry (ALG) measured in Newtons across all participants for baseline responses of the forearms to applied pressure showed significantly lower levels detected as painful by the paretic versus the unaffected arm (paired t-test: $\mathrm{t}_{(21)}=2 \cdot 3 ; p=0 \cdot 03$ ). Repeated measures from baseline (PRE), visit 1 (V1) and visit 2 (V2) after treatment were compared for changes between groups (Fig. 4A and B for the paretic and unaffected arm, respectively).

\section{Insert Figure 4 here.}

During the trial, a mean increase in the applied pressure (Newtons, N) was required to induce pain from the paretic arm of the control group, whereas a decrease was demonstrated for the etanercept group (Fig. 4A) and analysis by generalized estimating equations demonstrated that the group*treatment interaction was significant $(p=0.02)$. Post-hoc analysis demonstrated that the mean pressure pain threshold (in Newtons) for the paretic arm within the control group at visit 2 after treatment was significantly greater than the mean level at baseline (paired t-test: $\mathrm{t}_{(11)}=2 \cdot 53$; $p=0 \cdot 028)$. Within the etanercept group, analysis of the unaffected arm also showed a decrease in the pressure pain threshold at visit 2 after treatment compared to baseline, although this was not significant (paired t-test: $\mathrm{t}_{(9)}=1 \cdot 58 ; \mathrm{p}=0.147$; Fig. 4B).

\section{Other Secondary Measures:}

No significant effects were detected for the between group differences in fatigue (FAS), depression (BDI) or sit-to-stand (STS) measures over the course of the trial (Table 2).

Insert Table 2 here.

Impairment from stroke in parameters including MOCA, STS and BDI were noted to be insufficient in our study groups in order to enable detection of any changes and hence, these 
parameters were not further assessed. The change in fatigue levels (FAS; out of 50) from baseline for the two patient groups showed a similar trending improvement based on the slopes of the graphs (Fig. 5).

Insert Figure 5 here.

\section{Thermosensory analysis:}

Approximately $40 \%$ of patients with CPSP reportedly experience hypoalgesia [51]. Consistent with this observation, 7 of the 10 etanercept and 5 of the 12 control group (totalling $n=12$ out of the 22 participants on trial) showed thermal pain insensitivity at baseline with extensive thermal hypoalgesia displayed by the paretic arm (Supplementary Table 2). Using the thermosensory analyser, reduced sensitivity to perceive stimuli as either hot $(p<0.001)$ or cold $(p<0.001)$, as well as lower hot or cold pain thresholds (both $p<0.001$ ) were detected when comparing the paretic to the unaffected arm across all the participants at baseline.

\section{Insert Figure 6 here.}

Apart from a trend towards an increase in cold pain threshold over time in the etanercept group (Fig. 6; generalized estimating equations: group*treatment interaction, $\mathrm{p}=0.053$ ), no other significant differences were apparent in thermal detection or pain thresholds between the active and control groups. Etanercept within group bivariate analysis showed a significant correlation (Pearson's $\mathrm{r}=0.730 ; p=0.016$ ) in the magnitude of change from baseline to visit 2 (after treatment) comparing the decrease in \% pain levels (by the 0-100 point vNPRS-FPS) with the increase in cold pain detection. No significant correlation was detected within the control group (r $=0 \cdot 045, p>0 \cdot 5)$. 
In summary, these results demonstrate that there was a rapid (by 30-60 minutes post-treatment) pain alleviation which was maintained over time, together with marked improvement in both active and passive mobility of the paretic arm following two doses of etanercept treatment.

\section{Discussion:}

This is the first double-blinded randomized controlled trial in CPSP demonstrating the significant effects of two doses of perispinal etanercept in reducing pain and improving mobility. The significant reduction in pain remained evident 30 days after trial enrolment and was not only statistically significant but exceeded the minimal clinically important difference or MCID [52] indicating the clinical relevance of such findings. The effects were demonstrated to occur rapidly (starting within 30-60 minutes) after the first treatment and were also complemented by improvements in mobility indicating a role for perispinal etanercept in improving the overall quality of life in CPSP.

Improvements in mechanical and thermal pain sensitivities also showed some interesting trends. Changes in functional sit-to-stand times or psychological measures were not significantly different between the two groups. Caution should be observed when interpreting the results of this trial. Although the overall responses for the etanercept group were clearly apparent with the observed lowering in mean pain levels reaching significance, universal improvement in the primary outcome was not achieved. Four of the ten etanercept group showed no or limited effects on their pain levels. However, in contrast, 3 in 10 of the etanercept group showed complete or almost complete resolution of CPSP after either the first or second of the two perispinal treatments. These results indicate that etanercept demonstrates variable responses on CPSP at the individual level. At present, the possible reasons for the significant reduction in pain levels for several of the patients 
from the perispinal etanercept group, but not by all patients in this group, is not clear. Possible reasons may relate to the extent and outcome of the post-stroke reorganization in the central nervous system and factors including lesion size, extent and severity of the stroke damage, and time elapsed since stroke. Further studies will be required to resolve these points.

Although the processes associated with the reduction in constant levels of pain in the stroke patients are not readily apparent, trends towards a normalization of mechanical and thermal sensitivities were also demonstrated. A previous study of CPSP treatment using deep brain stimulation reported similar improvements in pain associated with normalization of deficits in somatosensory perception, including thermal sensitivity [53]. The results for algometry and thermosensory analysis of CPSP patients obtained in the present study suggest that the changes in the etanercept group were trending towards normalization of somatosensory function occurring with increased thermal and pressure pain sensitivity, in line with the other trial outcomes of improved neuromuscular function.

Typically, normal pain thresholds are approximately $45^{\circ} \mathrm{C}$ and $10^{\circ} \mathrm{C}$ for hot and cold (Medoc Advanced Medical Systems, Ltd) respectively. Based on the Medoc thresholds and from comparing thermal responses of the paretic to the unaffected arm, almost half of the patients in each group demonstrated thermal hypoalgesia at baseline. Notably, the two patients with the strongest pain reduction in the etanercept group also showed the greatest normalization in their thermal and mechanical pain sensitivity, consistent with the increasing sensitivity associated with returning or restoration of neurosensory function. This was further confirmed by bivariate correlation when comparing the magnitude of the reduction in CPSP levels with the increase in thermal sensitivity to cold temperature related pain responses.

\section{The role of TNF in central post-stroke pain.}


The data from this trial further adds to the mounting evidence supporting a direct role within the central nervous system for TNF involvement in pain modulation [54] and specifically for neuropathic pain conditions [21] such as CPSP. The present outcomes from this randomized clinical trial also supports the previously reported observational data that showed positive clinical responses to TNF inhibition in patients with chronic stroke and associated improvements in moderate to severe disability $[31,32,35]$. Elevated TNF levels have not only been demonstrated in the cerebral spinal fluid of acute stage $[9,12,13,14,15,16,17,18]$ but also chronic post-stroke patients [19], as well as in patients with clinical depression [55], traumatic brain injury [19, 56], multiple sclerosis, dementia and probably a host of other neurological disorders, as reviewed in [7, 34, 37]. This raises the spectre of TNF having a major role not only in the stroke penumbra and acute phase of damage, but also impacting on the ensuing global inflammatory aspects affecting the wider range of normal brain function [57]. The improvement in clinical outcomes in our study for CPSP patients after treatment with a TNF inhibitor further implicates the role of TNF in this condition as well.

The underlying mechanism(s) at the molecular level responsible for the improvement in clinical outcomes in our study are challenging to define. TNFalpha has been recently shown to induce the increased expression of the TRP family of calcium-channel related thermal nociceptors [58] and this action of TNF would increase pain sensitivity via nociceptor sensitization $[58,59]$. TNF/TNF receptor signalling is also required for development and function of primary nociceptors in sensory neurons [60]. The alleviation of CPSP in the present study supports this mechanism as possibly underlying changes in the central nervous system in response to etanercept actions shown here and reported elsewhere [29, 61]. However, the improvements in somatosensory perception in both arms demonstrated after treatment with etanercept are more difficult to explain. Despite the reduction in CPSP in the group administered perispinal etanercept, our study did not demonstrate 
a clear improvement in thermal sensory thresholds, although there was a trend towards normalization of cold pain thresholds in the etanercept group which correlated significantly with the decrease in CPSP within this group. Hence, the gain in responsiveness to both peripheral pressure and thermal stimuli suggest a normalization/restoration was also occurring in somatosensory perception by the central nervous system, along with the reduced CPSP. The small sample size likely precluded detecting more highly significant differences in thermal and pressure sensitivity. TNF has a plethora of other roles in the central nervous system for regulating neuronal function, synaptic plasticity and neurotransmitter activity related to pain $[62,63,64,65]$ which may also underlie mechanisms associated with the persistence of CPSP and the effects of antiTNF detected here.

\section{TNF and neuromuscular function.}

In view of the above neurosensory aspects of TNF, understanding TNF's role in control of neuromuscular function is limited. An unprecedented finding of this trial was that the majority $(90 \%)$ of patients within the etanercept group showed significant rapid enhancements in both active and passive shoulder flexion ROM by their paretic arm. This change in movement was irrespective of effects on their pain levels, with three out of the ten patients in the etanercept group regaining their full use and complete 180 degrees of active shoulder flexion. This result indicates that a relaxation in the extent of spasticity was likely occurring in the arm muscles of patients treated with perispinal etanercept, improving both their active and passive control of movement. TNFalpha has previously been demonstrated to be associated with muscle atrophy, particularly during cachexia in diseases such as cancer [66], and increased TNF levels have been linked with skeletal muscle loss/atrophy as a common sequelae associated with individuals with chronic stroke symptoms [67], as well as sarcopenia [68]. The rapid improvement (after first treatment) in active 
shoulder flexion provides evidence that such sequelae may be reversible and points to TNF also being involved in regulating neuromuscular function. Hence, when the results with loss of pain, changes in sensory (thermal and pressure) perception and improved mobility are considered together, occurring almost immediately following first treatment (with no significant changes in the control group), the data provides the first confirmatory supportive evidence from a randomized parallel double blinded clinical trial for rapid and wide-ranging benefits achievable by treating stroke patients with perispinal etanercept. As the perispinal etanercept treatment has a low risk side-effect profile and is well tolerated, it would seem to offer advantages over the current use of sedative drugs such as opioids or gabapentanoids for treatment of pain in stroke patients $[69,70]$.

A limitation of the present study was that it did not improve all the secondary outcomes of the trial, most likely because of the small sample size (total $n=22$ ) which precluded sufficient power to detect statistically significant responses for some of these measures. In addition, the improvements in BDI, STS and FAS tests in both groups (Table 2) indicated that the control group may have experienced a placebo effect within these secondary parameters, possibly reflecting the control group patients' positive beliefs in obtaining some benefit on trial. Alternatively, this could also have been an effect from the saline control acting as a dilution factor after injection into the cerebrospinal venous system or may reflect the relatively short duration of the study with measures spanning over a four-week period. In this regard, placebo effects have been previously reported to occur in some pain trials $[71,72]$. A small effect was noted with a trend towards lower mean pain detected after first treatment within the control group, but this was not significant. A possible reason for this would be the self-realization of the control patients during the trial that they were not obtaining any obvious improvement. 
Several questions arise from this study with the intent of optimizing patient outcomes in the longer term, particularly relating to the underlying causes for the variable response rates, with some but not all our stroke patients showing dramatic improvements after perispinal etanercept. With further studies it may be possible to expand on the application of perispinal etanercept therapy by identifying more precisely the determining factors for those patients who show significant responses, as well as the longevity of their effects. Our study's focus was on chronic stroke patients (average of four to five years since stroke) and the primary end point was 30 days after the first treatment. Thus, the role of anti-TNF in earlier stages of stroke, or the longevity of treatment effects cannot be determined from our data, nor can we address the possibility of greater improvements that might be obtained with further perispinal etanercept treatments. Hence, dose optimization clearly needs to be established, including determining the best regimen, the dosing level to be delivered via the cerebrospinal venous system, the optimal timing of stage of stroke and the intervals between successive treatments. Larger trials would also allow the underlying mechanisms to be further evaluated, with our current trial being underpowered to detect changes in thermal or pressure sensitivity/pain, albeit with a trend towards normalization.

Despite the relatively small sample size, the effect size and power of this study was sufficiently adequate for the primary outcome measure of pain, as was predicted based on the previously reported open-label results in stroke using the vertical pain assessment score [31]. Hence, together with the presently documented outcomes from small total participant numbers, our results bode well for any follow-up trials. It is advisable that follow-up studies consider the possibility of the short-term placebo effects as seen with some of the secondary exploratory measures examined here. These effects would likely dissipate over longer term evaluation as patients in the control group undergo the self-realization that they are on the saline treatment, given a lack of significant effects on their pain levels, or on their functional mobility. 


\section{Conclusions:}

This randomized, double-blinded, controlled parallel trial design significantly improved health outcomes in CPSP, particularly for reducing average and worst daily pain levels after treatment with perispinal etanercept, but not in the control group. Improvements were also obtained with secondary outcome measures of pain and functional mobility. The reduced pain severity has provided significant on-going benefits for some patients, at least over the medium term offering several months of reprieve (noted from follow-up post-trial) and points to a key role played by TNF in the manifestation of CPSP. Arguably, the availability of perispinal etanercept therapy for chronic stroke should be given a higher priority, and encouragement offered promoting further studies to be undertaken so that this treatment gains wider recognition and the acceptance required by the regulatory authorities. Clearly, the above results have confirmed the efficacy of perispinal etanercept therapy for improving outcomes with chronic stroke. It also emphasizes the need for further studies to identify in detail how to better exploit this information for alleviating the suffering experienced by stroke victims and to avoid the presently used and often ineffective drugs currently in clinical practice with their higher associated health risks.

\section{Transparency section.}

Trial Registration: The trial and outline of the study protocol was registered on the Australian and New Zealand Clinical Trials Registration (ANZCTR) database (17 Dec 2015; ACTRN12615001377527) and assigned Universal Trial Number U1111-1174-3242.

Acknowledgements: The investigators acknowledge the support of all patients who participated in this study. Also, they thank Dr Coralie Graham, Director, SRTF for support. The support of Dr Ed Tobinick for training in perispinal delivery and input into the design of the study protocol for the trial is also acknowledged. 
Funding details: This work was supported and sponsored by a grant from the Stroke Recovery Trial Fund (SRTF); Horizon Accounting, 21 Russell Street, Toowoomba, Queensland 4350, as well as public donations. The funders had no role in study design, data collection and analysis, decision to publish, or preparation of the manuscript.

Declaration of financial and other interests. The authors have no financial or other conflicts of interest to declare regarding this study.

Author contributions: All authors agree to be accountable for all aspects of the work and were involved in the conception, design and implementation of the trial. The first and seventh authors analysed and interpreted the data and prepared the manuscript. All authors were involved in revising the content and final approval of the version to be published.

\section{Data sharing statement:}

The data collected for the study, including participant data and a data dictionary can be made available to other investigator researchers who provide a methodologically sound and approved proposal. Individual participant data that underlie the results reported in this article, after deidentification (text, tables, figures, and appendices) can be made available beginning 3 months and ending 36 months following article publication. Proposals should be directed to the corresponding author to gain access and requests must meet with investigator approval and a signed data access agreement must be in place.

\section{References:}

1. Treister AK, Hatch MN, Cramer SC, et al. Demystifying Poststroke Pain: From Etiology to Treatment. PM R. 2017 Jan;9(1):63-75. doi: 10.1016/j.pmrj.2016.05.015. PubMed PMID: 27317916; PubMed Central PMCID: PMCPMC5161714.

2. Oh H, Seo W. A Comprehensive Review of Central Post-Stroke Pain. Pain Manag Nurs. 2015 Oct;16(5):804-18. doi: 10.1016/j.pmn.2015.03.002. PubMed PMID: 25962545. 
3. Akyuz G, Kuru P. Systematic Review of Central Post Stroke Pain: What Is Happening in the Central Nervous System? Am J Phys Med Rehabil. 2016 Aug;95(8):618-27. doi: 10.1097/PHM.0000000000000542. PubMed PMID: 27175563.

4. Haroutounian S, Ford AL, Frey K, et al. How central is central poststroke pain? The role of afferent input in poststroke neuropathic pain: a prospective, open-label pilot study. Pain. 2018 Jul;159(7):1317-1324. doi: 10.1097/j.pain.0000000000001213. PubMed PMID: 29570507.

5. Mulla SM, Wang L, Khokhar R, et al. Management of Central Poststroke Pain: Systematic Review of Randomized Controlled Trials. Stroke. 2015 Oct;46(10):2853-60. doi: 10.1161/STROKEAHA.115.010259. PubMed PMID: 26359361.

6. Fang $M$, Zhong $L$, Jin X, et al. Effect of Inflammation on the Process of Stroke Rehabilitation and Poststroke Depression. Front Psychiatry. 2019;10:184. doi: 10.3389/fpsyt.2019.00184. PubMed PMID: 31031649; PubMed Central PMCID: PMCPMC6470379.

7. Tuttolomondo A, Pecoraro R, Pinto A. Studies of selective TNF inhibitors in the treatment of brain injury from stroke and trauma: a review of the evidence to date. Drug Des Devel Ther. 2014;8:2221-38. doi: 10.2147/DDDT.S67655. PubMed PMID: 25422582; PubMed Central PMCID: PMCPMC4232043.

8. Cevik O, Adiguzel Z, Baykal AT, et al. The apoptotic actions of platelets in acute ischemic stroke. Mol Biol Rep. 2013 Dec;40(12):6721-7. doi: 10.1007/s11033-013-2787-9. PubMed PMID: 24057255.

9. Zaremba J, Losy J. Early TNF-alpha levels correlate with ischaemic stroke severity. Acta Neurol Scand. 2001 Nov;104(5):288-95. PubMed PMID: 11696023.

10. Zaremba J, Skrobanski P, Losy J. Tumour necrosis factor-alpha is increased in the cerebrospinal fluid and serum of ischaemic stroke patients and correlates with the volume of evolving brain infarct. Biomed Pharmacother. 2001 Jun;55(5):258-63. PubMed PMID: 11428551.

11. Barone FC, Arvin B, White RF, et al. Tumor necrosis factor-alpha. A mediator of focal ischemic brain injury. Stroke. 1997 Jun;28(6):1233-44. doi: 10.1161/01.str.28.6.1233. PubMed PMID: 9183357.

12. Tarkowski E, Rosengren L, Blomstrand C, et al. Intrathecal release of pro- and anti-inflammatory cytokines during stroke. Clin Exp Immunol. 1997 Dec;110(3):492-9. doi: 10.1046/j.13652249.1997.4621483.x. PubMed PMID: 9409656; PubMed Central PMCID: PMCPMC1904815.

13. Zaremba J, Losy J. The levels of TNF-alpha in cerebrospinal fluid and serum do not correlate with the counts of the white blood cells in acute phase of ischaemic stroke. Folia Morphol (Warsz). 2001;60(2):91-7. PubMed PMID: 11407149.

14. Zaremba J. Contribution of tumor necrosis factor alpha to the pathogenesis of stroke. Folia Morphol (Warsz). 2000;59(3):137-43. PubMed PMID: 10974781.

15. Lambertsen KL, Finsen B, Clausen BH. Post-stroke inflammation-target or tool for therapy? Acta Neuropathol. 2019 May;137(5):693-714. doi: 10.1007/s00401-018-1930-z. PubMed PMID: 30483945; PubMed Central PMCID: PMCPMC6482288.

16. Lambertsen KL, Biber K, Finsen B. Inflammatory cytokines in experimental and human stroke. J Cereb Blood Flow Metab. 2012 Sep;32(9):1677-98. doi: 10.1038/jcbfm.2012.88. PubMed PMID: 22739623; PubMed Central PMCID: PMCPMC3434626.

17. Nguyen TV, Frye JB, Zbesko JC, et al. Multiplex immunoassay characterization and species comparison of inflammation in acute and non-acute ischemic infarcts in human and mouse brain tissue. Acta Neuropathol Commun. 2016 Sep 6;4(1):100. doi: 10.1186/s40478-016-0371-y. PubMed PMID: 27600707; PubMed Central PMCID: PMCPMC5011964.

18. Beridze M, Sanikidze T, Shakarishvili R, et al. Selected acute phase CSF factors in ischemic stroke: findings and prognostic value. BMC Neurol. 2011 Mar 30;11:41. doi: 10.1186/1471-2377-11-41. PubMed PMID: 21450100; PubMed Central PMCID: PMCPMC3078848. 
19. Kim JW, Park MS, Kim JT, et al. The Impact of Tumor Necrosis Factor-alpha and Interleukin-1beta Levels and Polymorphisms on Long-Term Stroke Outcomes. Eur Neurol. 2018;79(1-2):38-44. doi: 10.1159/000484599. PubMed PMID: 29161722.

20. Dziewulska D, Mossakowski MJ. Cellular expression of tumor necrosis factor a and its receptors in human ischemic stroke. Clin Neuropathol. 2003 Jan-Feb;22(1):35-40. PubMed PMID: 12617192.

21. Ignatowski TA, Spengler RN. Targeting tumor necrosis factor in the brain relieves neuropathic pain. World J Anesthesiol. 2018;7(2):10-19. doi: 10.5313/wja.v7.i2.10.

22. Ogawa N, Kawai H, Terashima T, et al. Gene therapy for neuropathic pain by silencing of TNFalpha expression with lentiviral vectors targeting the dorsal root ganglion in mice. PLoS One. 2014;9(3):e92073. doi: 10.1371/journal.pone.0092073. PubMed PMID: 24642694; PubMed Central PMCID: PMCPMC3958473.

23. Gerard E, Spengler RN, Bonoiu AC, et al. Chronic constriction injury-induced nociception is relieved by nanomedicine-mediated decrease of rat hippocampal tumor necrosis factor. Pain. 2015 Jul;156(7):1320-33. doi: 10.1097/j.pain.0000000000000181. PubMed PMID: 25851457; PubMed Central PMCID: PMCPMC4474806.

24. Sommer C, Schafers M, Marziniak M, et al. Etanercept reduces hyperalgesia in experimental painful neuropathy. J Peripher Nerv Syst. 2001 Jun;6(2):67-72. PubMed PMID: 11446385.

25. Sommer C, Schäfers M. Mechanisms of neuropathic pain: the role of cytokines. Drug Discovery Today: Disease Mechanisms. 2004 2004/12/01/;1(4):441-448. doi: https://doi.org/10.1016/j.ddmec.2004.11.018.

26. Uceyler $\mathrm{N}$, Sommer $\mathrm{C}$. Cytokine regulation in animal models of neuropathic pain and in human diseases. Neurosci Lett. 2008 Jun 6;437(3):194-8. doi: 10.1016/j.neulet.2008.03.050. PubMed PMID: 18403115.

27. Tobinick EL. Perispinal Delivery of CNS Drugs. CNS Drugs. 2016 Jun;30(6):469-80. doi: 10.1007/s40263-016-0339-2. PubMed PMID: 27120182; PubMed Central PMCID: PMCPMC4920856.

28. Griessenauer CJ, Raborn J, Foreman P, et al. Venous drainage of the spine and spinal cord: a comprehensive review of its history, embryology, anatomy, physiology, and pathology. Clin Anat. 2015 Jan;28(1):75-87. doi: 10.1002/ca.22354. PubMed PMID: 24677178.

29. LaMacchia ZM, Spengler RN, Jaffari M, et al. Perispinal injection of a TNF blocker directed to the brain of rats alleviates the sensory and affective components of chronic constriction injuryinduced neuropathic pain. Brain Behav Immun. 2019 Jul 31. doi: 10.1016/j.bbi.2019.07.036. PubMed PMID: 31376497.

30. Tobinick E. Immediate Resolution of Hemispatial Neglect and Central Post-Stroke Pain After Perispinal Etanercept: Case Report. Clin Drug Investig. 2019 Oct 22. doi: 10.1007/s40261-01900864-8. PubMed PMID: 31642048.

31. Tobinick E, Kim NM, Reyzin G, et al. Selective TNF inhibition for chronic stroke and traumatic brain injury: an observational study involving 629 consecutive patients treated with perispinal etanercept. CNS Drugs. 2012 Dec;26(12):1051-70. doi: 10.1007/s40263-012-0013-2. PubMed PMID: 23100196.

32. Ignatowski TA, Spengler RN, Dhandapani KM, et al. Perispinal etanercept for post-stroke neurological and cognitive dysfunction: scientific rationale and current evidence. CNS Drugs. 2014 Aug;28(8):679-97. doi: 10.1007/s40263-014-0174-2. PubMed PMID: 24861337; PubMed Central PMCID: PMCPMC4110406.

33. Gronseth GS, Messe SR. Practice advisory: Etanercept for poststroke disability: Report of the Guideline Development, Dissemination, and Implementation Subcommittee of the American Academy of Neurology. Neurology. 2016 Jun 7;86(23):2208-11. doi: 10.1212/WNL.0000000000002735. PubMed PMID: 27272034; PubMed Central PMCID: PMCPMC4898316. 
34. Tobinick E. Perispinal etanercept advances as a neurotherapeutic. Expert Rev Neurother. 2018 Jun;18(6):453-455. doi: 10.1080/14737175.2018.1468253. PubMed PMID: 29695205.

35. Tobinick E. Rapid improvement of chronic stroke deficits after perispinal etanercept: three consecutive cases. CNS Drugs. 2011 Feb;25(2):145-55. doi: 10.2165/11588400-000000000-00000. PubMed PMID: 21254790.

36. Tobinick E. Perispinal etanercept: a new therapeutic paradigm in neurology. Expert Rev Neurother. 2010 Jun;10(6):985-1002. doi: 10.1586/ern.10.52. PubMed PMID: 20518613.

37. Tobinick E. Perispinal etanercept for neuroinflammatory disorders. Drug Discov Today. 2009 Feb;14(3-4):168-77. doi: 10.1016/j.drudis.2008.10.005. PubMed PMID: 19027875.

38. Tobinick E. Perispinal etanercept produces rapid improvement in primary progressive aphasia: identification of a novel, rapidly reversible TNF-mediated pathophysiologic mechanism. Medscape J Med. 2008 Jun 10;10(6):135. PubMed PMID: 18679537; PubMed Central PMCID: PMCPMC2491668.

39. Tobinick E. Perispinal etanercept for treatment of Alzheimer's disease. Curr Alzheimer Res. 2007 Dec;4(5):550-2. PubMed PMID: 18220520.

40. Tobinick EL, Gross H. Rapid cognitive improvement in Alzheimer's disease following perispinal etanercept administration. J Neuroinflammation. 2008 Jan 9;5:2. doi: 10.1186/1742-2094-5-2. PubMed PMID: 18184433; PubMed Central PMCID: PMCPMC2211476.

41. Tobinick E, Davoodifar S. Efficacy of etanercept delivered by perispinal administration for chronic back and/or neck disc-related pain: a study of clinical observations in 143 patients. Curr Med Res Opin. 2004 Jul;20(7):1075-85. doi: 10.1185/030079903125004286. PubMed PMID: 15265252.

42. Chuang $\mathrm{LL}, \mathrm{Wu} \mathrm{CY}$, Lin $\mathrm{KC}$, et al. Relative and absolute reliability of a vertical numerical pain rating scale supplemented with a faces pain scale after stroke. Phys Ther. 2014 Jan;94(1):129-38. doi: 10.2522/ptj.20120422. PubMed PMID: 24029301.

43. Chiti G, Pantoni L. Use of Montreal Cognitive Assessment in patients with stroke. Stroke. 2014 Oct;45(10):3135-40. doi: 10.1161/STROKEAHA.114.004590. PubMed PMID: 25116881.

44. Fullerton KJ, McSherry D, Stout RW. Albert's test: a neglected test of perceptual neglect. Lancet. 1986 Feb 22;1(8478):430-2. doi: 10.1016/s0140-6736(86)92381-0. PubMed PMID: 2868349.

45. Mead G, Lynch J, Greig C, et al. Evaluation of fatigue scales in stroke patients. Stroke. 2007 Jul;38(7):2090-5. doi: 10.1161/STROKEAHA.106.478941. PubMed PMID: 17525397.

46. Champod AS, Gubitz GJ, Phillips SJ, et al. Clock Drawing Test in acute stroke and its relationship with long-term functional and cognitive outcomes. Clin Neuropsychol. 2019 Jul;33(5):817-830. doi: 10.1080/13854046.2018.1494307. PubMed PMID: 29985104.

47. Niesters $M$, Aarts $L$, Sarton E, et al. Influence of ketamine and morphine on descending pain modulation in chronic pain patients: a randomized placebo-controlled cross-over proof-ofconcept study. Br J Anaesth. 2013 Jun;110(6):1010-6. doi: 10.1093/bja/aes578. PubMed PMID: 23384733.

48. Nothnagel $\mathrm{H}$, Puta $\mathrm{C}$, Lehmann $\mathrm{T}$, et al. How stable are quantitative sensory testing measurements over time? Report on 10-week reliability and agreement of results in healthy volunteers. J Pain Res. 2017;10:2067-2078. doi: 10.2147/JPR.S137391. PubMed PMID: 28919806; PubMed Central PMCID: PMCPMC5587201.

49. Strangfeld A, Listing J, Herzer $P$, et al. Risk of herpes zoster in patients with rheumatoid arthritis treated with anti-TNF-alpha agents. JAMA. 2009 Feb 18;301(7):737-44. doi: 10.1001/jama.2009.146. PubMed PMID: 19224750.

50. Di Costanzo L, Ayala F, Megna M, et al. The risk of herpes zoster in the anti-TNF-alpha era: a case report and review of the literature. J Dermatol Case Rep. 2013 Mar 30;7(1):1-4. doi: 10.3315/jdcr.2013.1126. PubMed PMID: 23580906; PubMed Central PMCID: PMCPMC3622506. 
51. Keszler M, Gude T, Heckert K. Pain syndromes associated with cerebrovascular accidents. In: Freedman MK, Gehret JA, Young GW, et al., editors. Challenging Neuropathic Pain Syndromes: Elsevier; 2018. p. 155-165.

52. Bushnell C, Bettger JP, Cockroft KM, et al. Chronic Stroke Outcome Measures for Motor Function Intervention Trials: Expert Panel Recommendations. Circ Cardiovasc Qual Outcomes. 2015 Oct;8(6 Suppl 3):S163-9. doi: 10.1161/CIRCOUTCOMES.115.002098. PubMed PMID: 26515205; PubMed Central PMCID: PMCPMC5289112.

53. Pickering AE, Thornton SR, Love-Jones SJ, et al. Analgesia in conjunction with normalisation of thermal sensation following deep brain stimulation for central post-stroke pain. Pain. 2009 Dec 15;147(1-3):299-304. doi: 10.1016/j.pain.2009.09.011. PubMed PMID: 19833434; PubMed Central PMCID: PMCPMC2789248.

54. Hess A, Axmann $R$, Rech J, et al. Blockade of TNF-alpha rapidly inhibits pain responses in the central nervous system. Proc Natl Acad Sci U S A. 2011 Mar 1;108(9):3731-6. doi: 10.1073/pnas.1011774108. PubMed PMID: 21245297; PubMed Central PMCID: PMCPMC3048151.

55. Fasick V, Spengler RN, Samankan S, et al. The hippocampus and TNF: Common links between chronic pain and depression. Neurosci Biobehav Rev. 2015 Jun;53:139-59. doi: 10.1016/j.neubiorev.2015.03.014. PubMed PMID: 25857253.

56. Hasturk AE, Gokce EC, Yilmaz ER, et al. Therapeutic Evaluation of Tumor Necrosis Factor-alpha Antagonist Etanercept against Traumatic Brain Injury in Rats: Ultrastructural, Pathological, and Biochemical Analyses. Asian J Neurosurg. 2018 Oct-Dec;13(4):1018-1025. doi: 10.4103/ajns.AJNS_29_17. PubMed PMID: 30459860; PubMed Central PMCID: PMCPMC6208262.

57. Shi K, Tian DC, Li ZG, et al. Global brain inflammation in stroke. Lancet Neurol. 2019 Jul 8. doi: 10.1016/S1474-4422(19)30078-X. PubMed PMID: 31296369.

58. Meng J, Wang J, Steinhoff $M$, et al. TNFalpha induces co-trafficking of TRPV1/TRPA1 in VAMP1containing vesicles to the plasmalemma via Munc18-1/syntaxin1/SNAP-25 mediated fusion. Sci Rep. 2016 Feb 18;6:21226. doi: 10.1038/srep21226. PubMed PMID: 26888187; PubMed Central PMCID: PMCPMC4758037.

59. Marrone MC, Morabito A, Giustizieri M, et al. TRPV1 channels are critical brain inflammation detectors and neuropathic pain biomarkers in mice. Nat Commun. 2017 May 10;8:15292. doi: 10.1038/ncomms15292. PubMed PMID: 28489079; PubMed Central PMCID: PMCPMC5436240.

60. Wheeler MA, Heffner DL, Kim S, et al. TNF-alpha/TNFR1 signaling is required for the development and function of primary nociceptors. Neuron. 2014 May 7;82(3):587-602. doi: 10.1016/j.neuron.2014.04.009. PubMed PMID: 24811380; PubMed Central PMCID: PMCPMC4046273.

61. Lu SC, Chang YS, Kan HW, et al. Tumor necrosis factor-alpha mediated pain hypersensitivity through Ret receptor in resiniferatoxin neuropathy. Kaohsiung J Med Sci. 2018 Sep;34(9):494-502. doi: 10.1016/j.kjms.2018.04.008. PubMed PMID: 30173779.

62. Liu Y, Zhou LJ, Wang J, et al. TNF-alpha Differentially Regulates Synaptic Plasticity in the Hippocampus and Spinal Cord by Microglia-Dependent Mechanisms after Peripheral Nerve Injury. J Neurosci. 2017 Jan 25;37(4):871-881. doi: 10.1523/JNEUROSCI.2235-16.2016. PubMed PMID: 28123022; PubMed Central PMCID: PMCPMC5296781.

63. Chen SX, Liao GJ, Yao PW, et al. Calpain-2 Regulates TNF-alpha Expression Associated with Neuropathic Pain Following Motor Nerve Injury. Neuroscience. 2018 Apr 15;376:142-151. doi: 10.1016/j.neuroscience.2018.02.023. PubMed PMID: 29477696.

64. Maggio N, Vlachos A. Tumor necrosis factor (TNF) modulates synaptic plasticity in a concentration-dependent manner through intracellular calcium stores. J Mol Med (Berl). 2018 Oct;96(10):1039-1047. doi: 10.1007/s00109-018-1674-1. PubMed PMID: 30073573. 
65. Yao $\mathrm{P}, \mathrm{Wang} \mathrm{S}, \mathrm{Xin} \mathrm{W}$, et al. Upregulation of tumor necrosis factor-alpha in the anterior cingulate cortex contributes to neuropathic pain and pain-associated aversion. Neurobiology of Disease. 2019;130.

66. Patel HJ, Patel BM. TNF-alpha and cancer cachexia: Molecular insights and clinical implications. Life Sci. 2017 Feb 1;170:56-63. doi: 10.1016/j.Ifs.2016.11.033. PubMed PMID: 27919820.

67. Hafer-Macko CE, Yu S, Ryan AS, et al. Elevated tumor necrosis factor-alpha in skeletal muscle after stroke. Stroke. 2005 Sep;36(9):2021-3. doi: 10.1161/01.STR.0000177878.33559.fe. PubMed PMID: 16109906.

68. Thoma A, Lightfoot AP. NF-kB and Inflammatory Cytokine Signalling: Role in Skeletal Muscle Atrophy. Adv Exp Med Biol. 2018;1088:267-279. doi: 10.1007/978-981-13-1435-3_12. PubMed PMID: 30390256.

69. Fleet JL, Dixon SN, Kuwornu PJ, et al. Gabapentin dose and the 30-day risk of altered mental status in older adults: A retrospective population-based study. PLoS One. 2018;13(3):e0193134. doi: 10.1371/journal.pone.0193134. PubMed PMID: 29538407; PubMed Central PMCID: PMCPMC5851574.

70. Khodneva Y, Muntner P, Kertesz S, et al. Prescription Opioid Use and Risk of Coronary Heart Disease, Stroke, and Cardiovascular Death Among Adults from a Prospective Cohort (REGARDS Study). Pain Med. 2016 Mar;17(3):444-455. doi: 10.1111/pme.12916. PubMed PMID: 26361245; PubMed Central PMCID: PMCPMC6281131.

71. Carvalho C, Caetano JM, Cunha L, et al. Open-label placebo treatment in chronic low back pain: a randomized controlled trial. Pain. 2016 Dec;157(12):2766-2772. doi: 10.1097/j.pain.0000000000000700. PubMed PMID: 27755279; PubMed Central PMCID: PMCPMC5113234.

72. Vachon-Presseau E, Berger SE, Abdullah TB, et al. Brain and psychological determinants of placebo pill response in chronic pain patients. Nat Commun. 2018 Sep 12;9(1):3397. doi: 10.1038/s41467018-05859-1. PubMed PMID: 30209286; PubMed Central PMCID: PMCPMC6135815.

73. Klit H, Finnerup NB, Jensen TS. Central post-stroke pain: clinical characteristics, pathophysiology, and management. Lancet Neurol. 2009 Sep;8(9):857-68. doi: 10.1016/S1474-4422(09)70176-0. PubMed PMID: 19679277. 
Table 1. Baseline characteristics of patients on the stroke trial

\begin{tabular}{|c|c|c|c|}
\hline $\begin{array}{l}\text { Characteristic } \\
\text { Mean (SE) }\end{array}$ & $\begin{array}{c}\text { Etanercept } \\
25 \mathrm{mg} \\
(\mathrm{n}=10)\end{array}$ & $\begin{array}{l}\text { Saline control } \\
\qquad(\mathrm{n}=12)\end{array}$ & $\begin{array}{c}\text { Mean difference }(95 \% \mathrm{CI}) \\
p \text { value }(2 \text {-tailed) }\end{array}$ \\
\hline Age, $y$ & $57 \cdot 3(4 \cdot 95)$ & $61 \cdot 65(8 \cdot 66)$ & $-4 \cdot 35(-15 \cdot 3$ to $6 \cdot 6), p=0 \cdot 42$ \\
\hline Weight, kg & $77 \cdot 74(5 \cdot 73)$ & $85 \cdot 85(4 \cdot 86)$ & $7 \cdot 09(-22 \cdot 9$ to $6 \cdot 7) ; p=0 \cdot 266$ \\
\hline Gender M:F & $5: 5$ & $7: 5$ & \\
\hline $\begin{array}{l}\text { Years since } \\
\text { stroke }\end{array}$ & $4 \cdot 18(0 \cdot 72)$ & $4 \cdot 98(1 \cdot 15)$ & $-0 \cdot 8(-3 \cdot 43$ to $1 \cdot 82), p=0 \cdot 52$ \\
\hline $\begin{array}{c}\text { Average daily } \\
\text { pain } \\
\text { (vNPRS-FPS) }\end{array}$ & $68(3 \cdot 35)$ & $60 \cdot 42(4 \cdot 85)$ & $7 \cdot 58(-5 \cdot 18$ to $20 \cdot 35), p=0 \cdot 23$ \\
\hline $\begin{array}{l}\text { Worst daily } \\
\text { pain }\end{array}$ & $82 \cdot 5(3 \cdot 96)$ & $74 \cdot 58(3 \cdot 45)$ & $7 \cdot 92(-3 \cdot 0$ to $18 \cdot 83), p=0 \cdot 15$ \\
\hline $\begin{array}{c}\text { Active } \\
\text { Shoulder } \\
\text { Flexion/ROM } \uparrow\end{array}$ & $55(15 \cdot 69)$ & $84 \cdot 11(13 \cdot 26)$ & $-29 \cdot 11(-73$ to $14 \cdot 8), p=0 \cdot 179$ \\
\hline $\begin{array}{c}\text { Passive } \\
\text { Shoulder } \\
\text { Flexion/ROM } \uparrow \\
\end{array}$ & $103(10 \cdot 25)$ & $116 \cdot 11(12 \cdot 35)$ & $\begin{array}{c}-13 \cdot 11(-46 \cdot 73 \text { to } 20 \cdot 51), p= \\
0 \cdot 42\end{array}$ \\
\hline MOCA & $21 \cdot 8(2 \cdot 33)$ & $25 \cdot 5(0 \cdot 77)$ & $-3 \cdot 66(-8 \cdot 6$ to $1 \cdot 29), p=0 \cdot 138$ \\
\hline BDI & $17 \cdot 6(3 \cdot 32)$ & $20 \cdot 8(3 \cdot 36)$ & $\begin{array}{c}-3 \cdot 23(-13 \cdot 18 \text { to } 6 \cdot 72), p= \\
0 \cdot 51\end{array}$ \\
\hline FAS & $31 \cdot 4(1 \cdot 54)$ & $34 \cdot 8(1 \cdot 62)$ & $\begin{array}{c}-3 \cdot 43(-8 \cdot 16 \text { to } 1 \cdot 29), p= \\
0 \cdot 145\end{array}$ \\
\hline STS & $19 \cdot 5(2 \cdot 7)$ & $24 \cdot 4(3 \cdot 5)$ & $-4 \cdot 88(-14 \cdot 4$ to $4 \cdot 6) ; p=0 \cdot 298$ \\
\hline CDT & N/A & N/A & N/A \\
\hline $\begin{array}{c}\text { Albert's Line } \\
\text { Bisection Test/ }\end{array}$ & N/A & N/A & N/A \\
\hline
\end{tabular}

Abbreviations: $\mathrm{SE}=$ standard error; $\mathrm{y}=$ years; $v$ NPRS-FPS $=$ vertical Numeric Pain Rating Scale with a Faces Pain Scale (0-100); MOCA = Montreal Cognitive Assessment test; BDI = Beck's Depression Inventory; STS $=5 \mathrm{x}$ Sit-to-Stand/seconds; FAS $=$ fatigue assessment score $/ 50$. CDT $=$ Clock Drawing Test. $\uparrow$ Values shown for patients with restricted $(<180$ degree $)$ shoulder flexion/rotation of movement (ROM) in arc degrees at baseline only for the paretic/hemiplegic arm ( $\mathrm{n}=10$ in etanercept group, $\mathrm{n}=9$ in control group). N/A: not applicable. 
TABLE 2. Secondary Outcomes: differences in test scores at Visit 2 after treatment compared with baseline.

\begin{tabular}{|l|l|l|l|l|}
\hline $\begin{array}{l}\text { Assessment/ } \\
\text { Out of total } \\
\text { score }\end{array}$ & \multicolumn{2}{|c|}{ Group Statistics } & \multicolumn{1}{|c|}{$\begin{array}{c}\text { Independent samples t-test for equality of } \\
\text { the means }\end{array}$} \\
\cline { 2 - 5 } & $\begin{array}{l}\text { Etanercept } \\
(\mathrm{n}=10) \text { (SE) }\end{array}$ & $\begin{array}{l}\text { Saline control } \\
(\mathrm{n}=12)(\mathrm{SE})\end{array}$ & $\begin{array}{c}\text { Mean Difference } \\
(95 \% \mathrm{CI})\end{array}$ & \multicolumn{1}{c|}{$\begin{array}{c}p \text { value } \\
\text { two-tailed) }\end{array}$} \\
\hline BDI / 63 & $-3 \cdot 8(1 \cdot 23)$ & $-6 \cdot 67(2 \cdot 46)$ & $2 \cdot 87(-2 \cdot 96$ to $8 \cdot 7)$ & $0 \cdot 31$ \\
\hline MOCA / 30 & $-0 \cdot 6(1 \cdot 79)$ & $\begin{array}{l}0 \cdot 55(0 \cdot 9) \\
(\mathrm{n}=11)\end{array}$ & $0 \cdot 56(-5 \cdot 22$ to $2 \cdot 93)$ & $0 \cdot 56$ \\
\hline FAS / 50 & $-4 \cdot 3(1 \cdot 35)$ & $-5 \cdot 8(1 \cdot 82)$ & $0 \cdot 78(-4 \cdot 12$ to $5 \cdot 69)$ & $0 \cdot 74$ \\
\hline STS/seconds & $-9 \cdot 2(2 \cdot 37)$ & $-9 \cdot 04(2 \cdot 64)$ & $0 \cdot 16(-7 \cdot 71$ to $7 \cdot 4)$ & $0 \cdot 97$ \\
\hline $\begin{array}{l}\text { ALG AFF } \\
\text { ARM/ N }\end{array}$ & $-2 \cdot 1(3 \cdot 9)$ & $10 \cdot 7(4 \cdot 23)$ & $-12 \cdot 8(-25$ to $-0 \cdot 6)$ & $0 \cdot 04$ \\
\hline $\begin{array}{l}\text { ALG GOOD } \\
\text { ARM/ N }\end{array}$ & $-7 \cdot 81(4 \cdot 91)$ & $1 \cdot 12(5 \cdot 89)$ & $-8 \cdot 93(-25 \cdot 35$ to $7 \cdot 5)$ & $0 \cdot 27$ \\
\hline
\end{tabular}

a. At day 30 compared to baseline. Abbreviations: ALG: Algometry of paretic/hemiplegic (affected; AFF) versus good arms measured in Newtons (N); BDI: Beck's Depression Inventory; MOCA: Montreal Cognitive Assessment; FAS: Fatigue Assessment Score; STS: Five times SitTo-Stand. 


\section{Figure Legends.}

Figure 1. Trial profile. Outline of study protocol and regimen for randomization on enrolment into the etanercept and control group, including numbers of patients in brackets and all dropouts due to exclusion or adverse events. ${ }^{*} \mathrm{D}$ : decision point upon trial completion after interim unblinding and primary outcome measures showed significance values for $p<0.05$.

Figure 2.A) Pain levels are rapidly decreased following PSE treatment. Mean changes in \% levels of average pain on the vNPRS-FPS (0-100 point scale) comparing between the saline control $(\times)$ and etanercept $(\diamond)$-treated groups \pm S.E. PRE: baseline; V1: Visit 1 after treatment 1; V2: Visit 2 after treatment 2; D30: day 30 after Visit 1. B) Box plot analysis of differences in median of worst and average pain levels comparing between baseline and day 30. Control group compared to perispinal etanercept (PSE) treatment group with medians, quartile ranges and outliers for changes in $\%$ pain levels as shown. ${ }^{*} p<0 \cdot 05$.

Figure 3. Rapid and marked improvement in active shoulder flexion by paretic arm after PSE treatment. Goniometry for mean changes in rotated angle (arc degrees) of A) active and B) passive shoulder flexion from baseline, comparing between the control $(\times)$ and etanercept $(\diamond)$ treated groups $( \pm$ S.E). PRE: baseline; V1: Visit 1 after treatment 1; V2: Visit 2 after treatment. ** $p<0 \cdot 01$.

Figure 4. Change in pressure sensitivity by algometry. Comparison of etanercept versus control treatment groups for sensitivity to applied pressure by algometry on the A) paretic/hemiplegic arm versus B) unaffected arm. Mean change shown for the control $(\times)$ and etanercept $(\diamond)$-treated groups ( \pm S.E). PRE: baseline; V1: Visit 1 after treatment 1; V2: Visit 2 after treatment. $p>0 \cdot 05$. Scale for applied pressure measured in Newtons $(\mathrm{N})$.

Figure 5. Similar change in Fatigue Assessment Scores (FAS). Both control $(\times)$ and etanercept $(\diamond)$ groups $( \pm$ S.E) showed improvement in mean fatigue levels (lower values out of total 50) over the trial period. PRE: baseline; V2: Visit 2 after treatment 2; D30: day 30 after Visit 1. $p>0 \cdot 05$.

Figure 6. Quantitative thermosensory analysis. Change in recorded temperature $\left({ }^{0} \mathrm{C}\right)$ for cold pain sensitivity of control $(\times)$ versus etanercept $(\diamond)$ groups $( \pm$ S.E) during trial. PRE: baseline; V1: Visit 1 after treatment; V2: Visit 2 after treatment. $p>0 \cdot 05$. 


\section{Supplementary Table 1.}

\section{Complete List of Specific Inclusion Criteria:}

1) Age between $30-80$ years old.

2) Stroke that occurred at least 6 months and not more than 15 years prior to screening for this study;

3) Chronic neurological impairment, including hemiparesis, following an ischemic stroke in the territory of the middle cerebral arteries (MCA) (including MCA clot or embolus or carotid occlusion causing MCA territory stroke) or basal ganglia form of intracerebral haemorrhage.

4) Chronic Post-Stroke Pain with the following characteristics:

-History of stroke and onset of pain at or after stroke onset;

- Confirmation of stroke by radiologist report (MRI or CT imaging);

-The CPSP or discomfort has been present daily for at least 14 hours per day for at least the past 3 months;

- Current and previous drug treatment for the pain is inadequate;

-The CPSP is moderate-to-severe in intensity, with a daily average intensity between $4-8$ on an 11-point (1-10) vertical numerical pain rating scale (vNPRS);

-The CPSP is in an area of the body affected by the stroke, i.e. side of the body (e.g. the left head, scalp, face, trunk, shoulder, arm, hand, leg, or foot).

-The CPSP includes chronic post-stroke pain involving the shoulder.

5) A component of the Chronic Post-Stroke Pain has characteristics suggestive of Central PostStroke Pain (CPSP), such as:

-The pain is associated with sensory loss and/or sensory hypersensitivity (dysesthesia), including: allodynia, hypoesthesia to touch or temperature, paresthesia, or hyper/hypoalgesia;

-The pain occurs while at rest, even without movement;

-The subject uses descriptors to describe the pain such as "burning", "painful cold", "electric shocks", "aching", "stinging", "pressing" or "pins and needles".

-The subject meets at least $4 / 5$ criteria of the grading system for CPSP for a component of their pain [73], as assessed by the study neurologist.

6) Subject is able to ambulate at least 5 meters (with or without a cane or walker) without assistance from another individual.

\section{Exclusion Criteria:}

1. Dementia diagnosis prior to date of stroke

2. Brain stem or cerebellar stroke

3. More than one stroke in the past 3 years

4. Parkinson's disease or Parkinsonian symptoms

5. Dementia with Lewy bodies

6. Multiple sclerosis at present or in the past

7. Demyelinating disease at present or in the past

8. History of tuberculosis

9. Positive PPD

10. HIV infection

11. History of hepatitis B

12. History of deep fungal infection (coccidiodomycosis, histoplasmosis, blastomycosis)

13. Active infection

14. Indwelling urinary catheter 
15. Lymphoma, active or in the past

16. Cancer within the past 5 years, non-melanoma skin cancer excluded

17. History of malignant melanoma

18. Uncontrolled diabetes mellitus

19. Subjects using any immunosuppressive medication, including Kineret (Anakinra) or Abatacept, or glucocorticoids currently.

20. Use of a TNF inhibitor (etanercept, infliximab, etc.) in the past

21. Congestive Heart Failure

22. Non-ambulatory

23. Less than two months since hospitalization for any cause

24. Pregnancy or breast-feeding

25. Psychosis or use of anti-psychotic medication (e.g. olanzapine, quetiapine, clozapine, aripiprazole, haloperidol, flupenazine, risperidone, ziprasidone)

26. History of alcohol abuse within 1 year of study entry

27. Autoimmune disorder

28. Previous neck surgery (such as cervical fusion)

29. Severe or unstable concomitant condition or disease (e.g., known significant neurologic deficit, cancer, hematologic, or coronary disease), or chronic condition (e.g., psychiatric disorder), which, in the opinion of the investigator, would affect the assessment of the safety and tolerability of perispinal etanercept.

30. Severe aphasia.

31. Mini-mental standard examination (MMSE) less than 16.

32. Subject is not using anti-coagulant (e.g. warfarin, etc.) or anti-platelet drug treatment (e.g. aspirin, etc.) to reduce the risk of ischemic stroke.

33. Subarachnoid hemorrhage

34. New pain drug added or previous pain drug stopped during the 14 days preceding study enrolment.

35. Body mass index greater than 40 .

36. Grand mal seizure within 3 months of study enrolment.

37. Subject has significant chronic pain other than chronic post-stroke pain that will, in the opinion of the screening neurologist, interfere with the evaluation of the response of the PSP to treatment.

38. Subject has received any investigational drug within $30 \mathrm{~d}$ before screening, or is scheduled to receive an investigational drug, other than the blinded-study drug during the course of this study. 39. Chronic shoulder pain preceding the stroke. 


\section{Supplementary Table 2: Median [IQR] thermal pain detection and pain thresholds $\left({ }^{\circ} \mathrm{C}\right)^{\mathrm{a}}$}

\begin{tabular}{|c|c|c|c|c|c|c|c|c|c|}
\hline \multirow{3}{*}{\multicolumn{2}{|c|}{ Group }} & \multicolumn{4}{|c|}{ Cold Perception Threshold } & \multicolumn{4}{|c|}{ Heat Perception Threshold } \\
\hline & & \multicolumn{2}{|c|}{ Perispinal Etanercept } & \multicolumn{2}{|c|}{ Saline Control } & \multicolumn{2}{|c|}{ Perispinal Etanercept } & \multicolumn{2}{|c|}{ Saline Control } \\
\hline & & Paretic & \multirow[t]{2}{*}{ Unaffected } & \multirow[t]{2}{*}{ Paretic } & \multirow{2}{*}{ Unaffected } & \multirow[t]{2}{*}{ Paretic } & \multirow[t]{2}{*}{ Unaffected } & \multirow[t]{2}{*}{ Paretic } & \multirow{2}{*}{ Unaffected } \\
\hline Visit & Treatment & atentic & & & & & & & \\
\hline 1 & PRE & $\begin{array}{l}16 \cdot 8 \\
{[9 \cdot 7} \\
24 \cdot 7]\end{array}$ & $\begin{array}{l}29 \cdot 1 \\
{[24 \cdot 8,} \\
30 \cdot 3]\end{array}$ & $\begin{array}{l}23 \cdot 2 \\
{[16 \cdot 4} \\
27 \cdot 9]\end{array}$ & $\begin{array}{l}26 \cdot 2 \\
{[25 \cdot 4,} \\
29 \cdot 8]\end{array}$ & $\begin{array}{l}46 \cdot 0 \\
{[37 \cdot 2,} \\
48 \cdot 0]\end{array}$ & $\begin{array}{c}35 \cdot 1 \\
{[34 \cdot 3} \\
38 \cdot 7]\end{array}$ & $\begin{array}{c}37 \cdot 7 \\
{[34 \cdot 7} \\
43 \cdot 2]\end{array}$ & $\begin{array}{c}35 \cdot 8 \\
{[34 \cdot 1} \\
38 \cdot 1]\end{array}$ \\
\hline & Post & $\begin{array}{c}18 \cdot 3 \\
{[12 \cdot 8,27 \cdot 2}\end{array}$ & $\begin{array}{r}28 \cdot 6 \\
{[25 \cdot 6,} \\
29 \cdot 8]\end{array}$ & $\begin{array}{c}26 \cdot 0 \\
{[20 \cdot 5} \\
27 \cdot 6]\end{array}$ & $\begin{array}{c}28 \cdot 4 \\
{[26 \cdot 8} \\
29 \cdot 8]\end{array}$ & $\begin{array}{l}44 \cdot 1 \\
{[37 \cdot 7} \\
45 \cdot 7]\end{array}$ & $\begin{array}{r}34 \cdot 6 \\
{[34 \cdot 0,} \\
37 \cdot 6]\end{array}$ & $\begin{array}{c}38 \cdot 3 \\
{[35 \cdot 0} \\
43 \cdot 2] \\
\end{array}$ & $\begin{array}{r}36 \cdot 7 \\
{[34 \cdot 3} \\
39 \cdot 9] \\
\end{array}$ \\
\hline 2 & PRE & $\begin{array}{c}24 \cdot 9 \\
{[12 \cdot 1} \\
27 \cdot 8]\end{array}$ & $\begin{array}{l}26 \cdot 6 \\
{[12 \cdot 6,} \\
29 \cdot 9]\end{array}$ & $\begin{array}{l}26 \cdot 8 \\
{[19 \cdot 7} \\
28 \cdot 5]\end{array}$ & $\begin{array}{c}29 \cdot 4 \\
{[27 \cdot 6} \\
30 \cdot 1]\end{array}$ & $\begin{array}{c}39 \cdot 4 \\
{[35 \cdot 0,} \\
46 \cdot 5]\end{array}$ & $\begin{array}{c}35 \cdot 6 \\
{[34 \cdot 4} \\
40 \cdot 7]\end{array}$ & $\begin{array}{c}36 \cdot 7 \\
{[35 \cdot 4} \\
45 \cdot 4]\end{array}$ & $\begin{array}{r}35 \cdot 1 \\
{[33 \cdot 5} \\
37 \cdot 3]\end{array}$ \\
\hline & Post & $\begin{array}{l}21 \cdot 5 \\
{[4 \cdot 6} \\
24 \cdot 9]\end{array}$ & $\begin{array}{l}28 \cdot 5 \\
{[27 \cdot 6} \\
29 \cdot 7]\end{array}$ & $\begin{array}{l}23 \cdot 4 \\
{[17 \cdot 1} \\
27 \cdot 6]\end{array}$ & $\begin{array}{l}27 \cdot 8 \\
{[26 \cdot 6} \\
28 \cdot 8]\end{array}$ & $\begin{array}{r}40 \cdot 4 \\
{[37 \cdot 6} \\
46 \cdot 3]\end{array}$ & $\begin{array}{c}34 \cdot 8 \\
{[34 \cdot 4} \\
35 \cdot 5]\end{array}$ & $\begin{array}{r}36 \cdot 2 \\
{[34 \cdot 5} \\
44 \cdot 6]\end{array}$ & $\begin{array}{r}35 \cdot 8 \\
{[34 \cdot 7} \\
38 \cdot 3]\end{array}$ \\
\hline
\end{tabular}

a. Values as shown are the temperature medians and [interquartile ranges (IQR)]

Thermal Pain Thresholds $\left({ }^{\circ} \mathrm{C}\right)$

\begin{tabular}{|c|c|c|c|c|c|c|c|c|c|}
\hline & & \multicolumn{4}{|c|}{ Cold Pain Threshold } & \multicolumn{4}{|c|}{ Heat Pain Threshold } \\
\hline \multirow{2}{*}{\multicolumn{2}{|c|}{$\begin{array}{c}\text { Group } \\
\text { Arm Tested }\end{array}$}} & \multicolumn{2}{|c|}{ Perispinal Etanercept } & \multicolumn{2}{|c|}{ Saline Control } & \multicolumn{2}{|c|}{ Perispinal Etanercept } & \multicolumn{2}{|c|}{ Saline Control } \\
\hline & & \multirow[t]{2}{*}{ Paretic } & \multirow[t]{2}{*}{ Unaffected } & \multirow[t]{2}{*}{ Paretic } & \multirow[t]{2}{*}{ Unaffected } & \multirow[t]{2}{*}{ Paretic } & \multirow[t]{2}{*}{ Unaffected } & \multirow{2}{*}{ Paretic } & \multirow{2}{*}{ Unaffected } \\
\hline Visit & Treatment & & & & & & & & \\
\hline \multirow[t]{2}{*}{1} & PRE & $\begin{array}{c}5 \cdot 5 \\
{[3 \cdot 8,} \\
12 \cdot 1]\end{array}$ & $\begin{array}{c}10 \cdot 8 \\
{[4 \cdot 9,20 \cdot 9]}\end{array}$ & $\begin{array}{l}12 \cdot 0 \\
{[3 \cdot 6} \\
24 \cdot 3]\end{array}$ & $\begin{array}{c}15 \cdot 4 \\
{[7 \cdot 1,23 \cdot 9]}\end{array}$ & $\begin{array}{c}48 \cdot 6 \\
{[46 \cdot 4,} \\
49 \cdot 3]\end{array}$ & $\begin{array}{c}46 \cdot 4 \\
{[39 \cdot 6,} \\
48 \cdot 1]\end{array}$ & $\begin{array}{c}47 \cdot 2 \\
{[44 \cdot 8,} \\
47 \cdot 8]\end{array}$ & $\begin{array}{r}43 \cdot 9 \\
{[41 \cdot 5} \\
47 \cdot 1]\end{array}$ \\
\hline & Post & $\begin{array}{c}8 \cdot 3 \\
{[5 \cdot 8,16 \cdot 3}\end{array}$ & $\begin{array}{l}16 \cdot 3 \\
{[15 \cdot 5} \\
21 \cdot 4]\end{array}$ & $\begin{array}{l}12 \cdot 4 \\
{[5 \cdot 7,} \\
24 \cdot 8]\end{array}$ & $\begin{array}{c}20 \cdot 6 \\
{[10 \cdot 5} \\
25 \cdot 8]\end{array}$ & $\begin{array}{c}48 \cdot 2 \\
{[46 \cdot 8,} \\
49 \cdot 1]\end{array}$ & $\begin{array}{c}44 \cdot 6 \\
{[39 \cdot 4,} \\
47 \cdot 1]\end{array}$ & $\begin{array}{l}46 \cdot 5 \\
{[42 \cdot 9} \\
48 \cdot 3]\end{array}$ & $\begin{array}{l}45 \cdot 5 \\
{[39 \cdot 5,} \\
47 \cdot 3]\end{array}$ \\
\hline 2 & PRE & $8 \cdot 4$ & $\begin{array}{c}12 \cdot 1 \\
{[4 \cdot 4,17 \cdot 1]}\end{array}$ & $13 \cdot 0$ & $\begin{array}{c}15 \cdot 7 \\
{[8 \cdot 4,26 \cdot 0]}\end{array}$ & $49 \cdot 1$ & $44 \cdot 9$ & $47 \cdot 7$ & $45 \cdot 4$ \\
\hline
\end{tabular}




\begin{tabular}{|c|c|c|c|c|c|c|c|c|c|}
\hline & {$[2 \cdot 9$,} & & {$[5 \cdot 4$,} & & {$[45 \cdot 1$,} & {$[42 \cdot 8$,} & {$[43 \cdot 7$,} & {$[39 \cdot 8$,} \\
& $18 \cdot 2]$ & & $25 \cdot 6]$ & & $49 \cdot 5]$ & $46 \cdot 5]$ & $48 \cdot 4]$ & $47 \cdot 7]$ \\
\cline { 2 - 8 } & \multirow{2}{*}{ Post } & $10 \cdot 4$ & $16 \cdot 5$ & $9 \cdot 9$ & $18 \cdot 6$ & $48 \cdot 4$ & $43 \cdot 8$ & $47 \cdot 4$ & $43 \cdot 8$ \\
& {$[4 \cdot 9$,} & {$[5 \cdot 4,16 \cdot 5]$} & {$[4 \cdot 8$,} & {$[8 \cdot 4,26 \cdot 1]$} & {$[45 \cdot 5$,} & {$[40 \cdot 8$,} & {$[45 \cdot 7$,} & {$[40 \cdot 0$,} \\
& $19 \cdot 1]$ & & $19 \cdot 8]$ & & $49 \cdot 2]$ & $47 \cdot 9]$ & $48 \cdot 0]$ & $46 \cdot 2]$ \\
\hline
\end{tabular}

For analysis of thermal perception and pain thresholds, multilevel modeling, using the linear mixed models (LMM) function in SPSS. When comparing the effect of active treatment on thermal thresholds, individual random intercept LMMs were constructed. The fixed effects of Visit (1 or 2), Treatment (PRE/Visit 1 after treatment/Visit 2 after treatment), Body Region (Pain Affected Arm/Unaffected Arm) and study Group (Etanercept/Control) were included in the model. The interaction between Treatment and Group (Treatment* Group) and Visit, Treatment and Group (Visit* Treatment* Group) were examined. Bonferroni corrections were applied for multiple comparisons and $p$-values $<0.05$ were considered significant.

For cold perception, a main effect of Body Region was detected with lower thresholds demonstrated on the paretic compared to the unaffected arm $(p<0 \cdot 001)$. This reflected a reduced sensitivity to cold on the paretic arm irrespective of group, visit number or treatment. Group did not interact with Treatment $(p=0 \cdot 72)$ or differentially influence the effects of Treatment at either Visit $(p=0 \cdot 76)$ on cold perception. No significant effects of Group $(p=0 \cdot 11)$, Visit $(p=0 \cdot 88)$ or Treatment $(p=0 \cdot 86)$ were detected on cold perception.

For heat perception, higher thresholds were demonstrated on the paretic arm $(p<0 \cdot 001)$, reflecting a reduced sensitivity to heat by this arm. Group did not interact with Treatment $(p=0 \cdot 35)$ or differentially influence the effect of Treatment at either Visit $(p=0 \cdot 35)$ on heat perception. No significant effects of Group $(p=1.00)$, Visit $(p=0 \cdot 22)$ or Treatment $(p=0 \cdot 75)$ were detected on heat perception.

For cold pain thresholds, lower thresholds were demonstrated on the paretic arm $(p<0 \cdot 001)$, reflecting reduced cold pain sensitivity at baseline. A main effect of Treatment $(p=0 \cdot 01)$, with higher pain thresholds (increased sensitivity to cold induced pain) was demonstrated, irrespective of Group or Visit. Group did not interact with Treatment $(p=0 \cdot 15)$ or differentially influence the effect of Treatment at either Visit $(p=0 \cdot 83)$ on cold pain perception. No significant effects of Group $(p=0 \cdot 26)$ or Visit $(p=0 \cdot 71)$ were detected for cold pain sensitivity.

For heat pain thresholds, higher thresholds were demonstrated on the affected arm $(p<0 \cdot 001)$, reflecting reduced heat pain sensitivity. Group did not interact with Treatment $(p=0 \cdot 74)$ or differentially influence the effect of Treatment at either Visit $(p=0 \cdot 93)$ on hot pain perceptions. No significant effects of Group $(p=0 \cdot 40)$, Visit $(p=0 \cdot 96)$ or Treatment $(p=0 \cdot 41)$ were detected for hot pain sensitivity. 
The effect of Group and Treatment on Sensory Lumen values (the difference between cold/heat pain thresholds and cold/heat pain perception) were examined to determine whether treatment 'narrowed' or 'widened' these lumens. The affected side demonstrated an increased sensory lumen ('widened') for both heat and cold, but this was irrespective of Group or Treatment status (results not shown). 
CONSORT 2010 checklist of information to include when reporting a randomised trial*

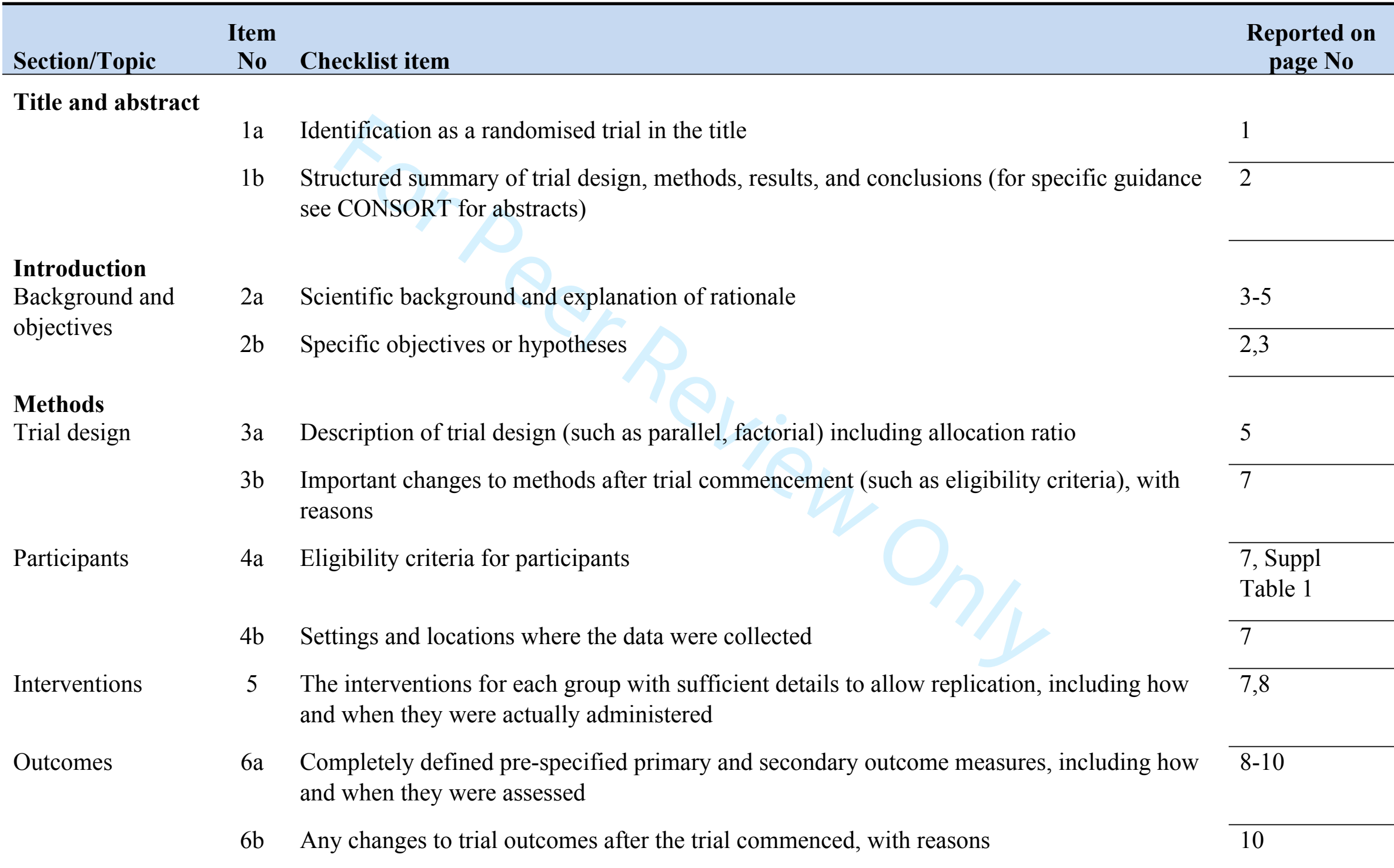


Sample size

Randomisation:

Sequence generation

Allocation concealment mechanism

Implementation

Blinding

Statistical methods

\section{Results}

Participant flow (a diagram is strongly recommended)

Recruitment 7a How sample size was determined

$7 \mathrm{~b}$ When applicable, explanation of any interim analyses and stopping guidelines

8a Method used to generate the random allocation sequence

$8 \mathrm{~b}$ Type of randomisation; details of any restriction (such as blocking and block size)

9 Mechanism used to implement the random allocation sequence (such as sequentially numbered containers), describing any steps taken to conceal the sequence until interventions were assigned

10 Who generated the random allocation sequence, who enrolled participants, and who assigned participants to interventions

11a If done, who was blinded after assignment to interventions (for example, participants, care providers, those assessing outcomes) and how

$11 \mathrm{~b}$ If relevant, description of the similarity of interventions

12a Statistical methods used to compare groups for primary and secondary outcomes

12b Methods for additional analyses, such as subgroup analyses and adjusted analyses

13a For each group, the numbers of participants who were randomly assigned, received intended treatment, and were analysed for the primary outcome

13b For each group, losses and exclusions after randomisation, together with reasons

14a Dates defining the periods of recruitment and follow-up

$14 \mathrm{~b}$ Why the trial ended or was stopped

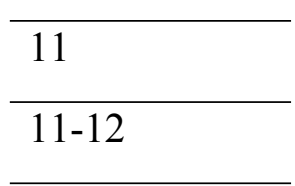

12

12

12

12

12

\begin{tabular}{l}
\hline 12 \\
\hline 12,13 \\
\hline 13
\end{tabular}

Fig. 1, p5

\begin{tabular}{l}
\hline 5,14 \\
\hline 14 \\
\hline 14 \\
\hline
\end{tabular}




\begin{tabular}{|c|c|c|c|}
\hline Baseline data & 15 & A table showing baseline demographic and clinical characteristics for each group & Table 1,15 \\
\hline Numbers analysed & 16 & $\begin{array}{l}\text { For each group, number of participants (denominator) included in each analysis and whether } \\
\text { the analysis was by original assigned groups }\end{array}$ & 15 \\
\hline \multirow[t]{2}{*}{$\begin{array}{l}\text { Outcomes and } \\
\text { estimation }\end{array}$} & $17 \mathrm{a}$ & $\begin{array}{l}\text { For each primary and secondary outcome, results for each group, and the estimated effect } \\
\text { size and its precision (such as } 95 \% \text { confidence interval) }\end{array}$ & $15-25$ \\
\hline & $17 \mathrm{~b}$ & For binary outcomes, presentation of both absolute and relative effect sizes is recommended & N/A \\
\hline Ancillary analyses & 18 & $\begin{array}{l}\text { Results of any other analyses performed, including subgroup analyses and adjusted analyses, } \\
\text { distinguishing pre-specified from exploratory }\end{array}$ & $20-25$ \\
\hline Harms & 19 & $\begin{array}{l}\text { All important harms or unintended effects in each group (for specific guidance see } \\
\text { CONSORT for harms) }\end{array}$ & 14 \\
\hline \multicolumn{4}{|l|}{ Discussion } \\
\hline Limitations & 20 & $\begin{array}{l}\text { Trial limitations, addressing sources of potential bias, imprecision, and, if relevant, } \\
\text { multiplicity of analyses }\end{array}$ & $21-26$ \\
\hline Generalisability & 21 & Generalisability (external validity, applicability) of the trial findings & $26-27$ \\
\hline Interpretation & 22 & $\begin{array}{l}\text { Interpretation consistent with results, balancing benefits and harms, and considering other } \\
\text { relevant evidence }\end{array}$ & $21-27$ \\
\hline \multicolumn{4}{|l|}{ Other information } \\
\hline Registration & 23 & Registration number and name of trial registry & 3 \\
\hline Protocol & 24 & Where the full trial protocol can be accessed, if available & 28 \\
\hline Funding & 25 & Sources of funding and other support (such as supply of drugs), role of funders & 27 \\
\hline
\end{tabular}

*We strongly recommend reading this statement in conjunction with the CONSORT 2010 Explanation and Elaboration for important clarifications on all the items. If relevant, we also recommend reading CONSORT extensions for cluster randomised trials, non-inferiority 
and equivalence trials, non-pharmacological treatments, herbal interventions, and pragmatic trials. Additional extensions are forthcoming: for those and for up to date references relevant to this checklist, see www.consort-statement.org. 
Figure 1: Trial profile

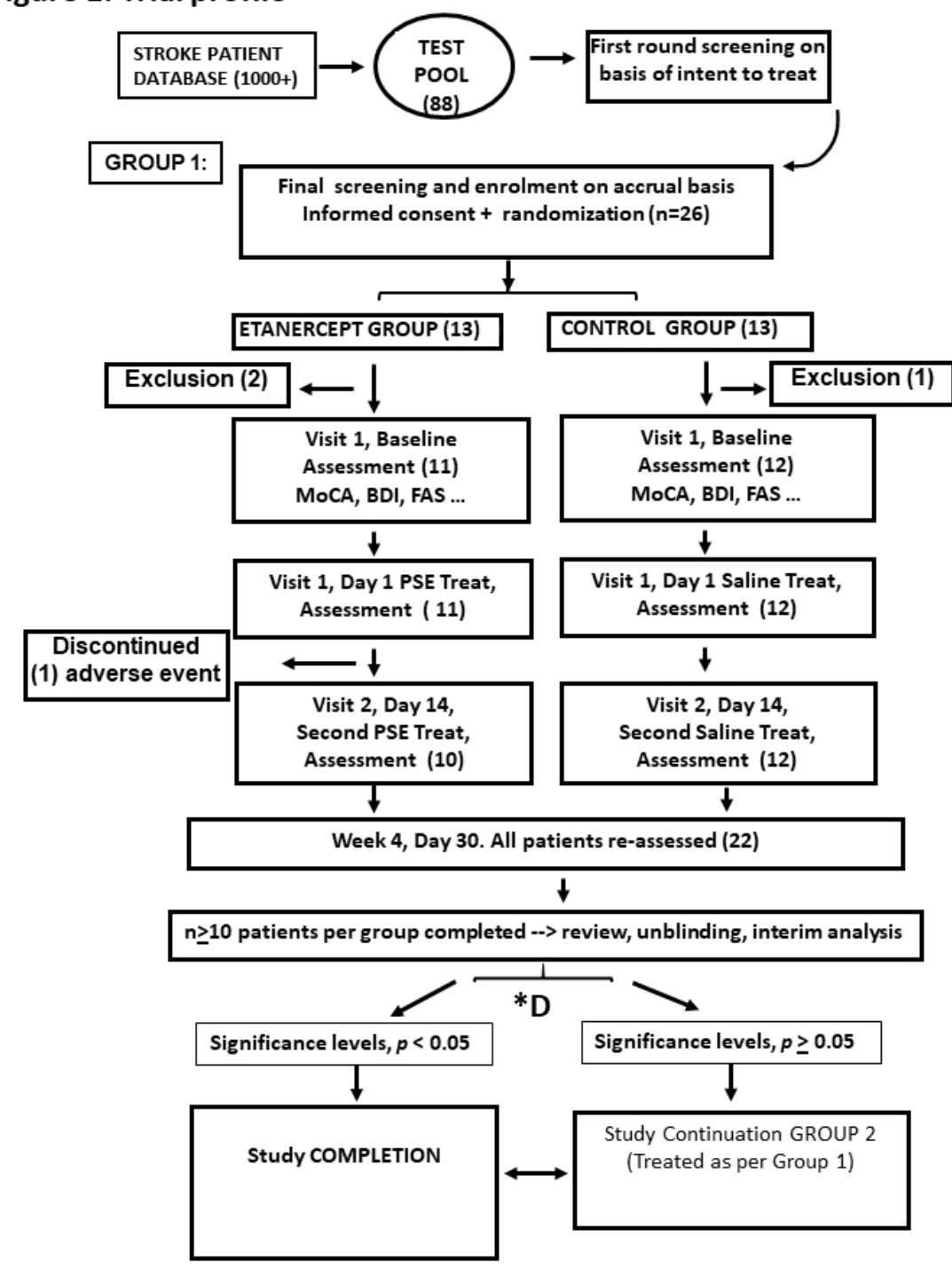

Figure 1. Trial profile. Outline of study protocol and regimen for randomization on enrolment into the etanercept and control group, including numbers of patients in brackets and all dropouts due to exclusion or adverse events. ${ }^{*} \mathrm{D}$ : decision point upon trial completion after interim unblinding and primary outcome measures showed significance values for $p<0.05$

$$
190 \times 254 \mathrm{~mm}(96 \times 96 \mathrm{DPI})
$$


Figure 2

Figure 2.A) Pain levels are rapidly decreased following PSE treatment. Mean changes in \% levels of average pain on the VNPRS-FPS (0-100 point scale) comparing between the saline control $(\times)$ and etanercept $(\diamond)$ treated groups \pm S.E. PRE: baseline; V1: Visit 1 after treatment 1; V2: Visit 2 after treatment 2; D30: day 30 after Visit 1. B) Box plot analysis of differences in median of worst and average pain levels comparing between baseline and day 30. Control group compared to perispinal etanercept (PSE) treatment group with medians, quartile ranges and outliers for changes in $\%$ pain levels as shown. $* p<0.05$.

$190 \times 254 \mathrm{~mm}(96 \times 96 \mathrm{DPI})$ 


\section{Figure 3}

\section{A) Change in active shoulder flexion}

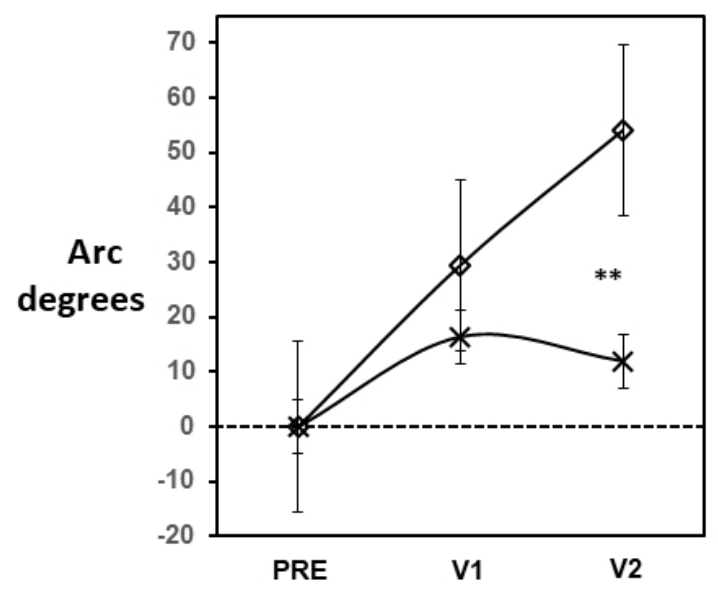

B) Change in passive shoulder flexion

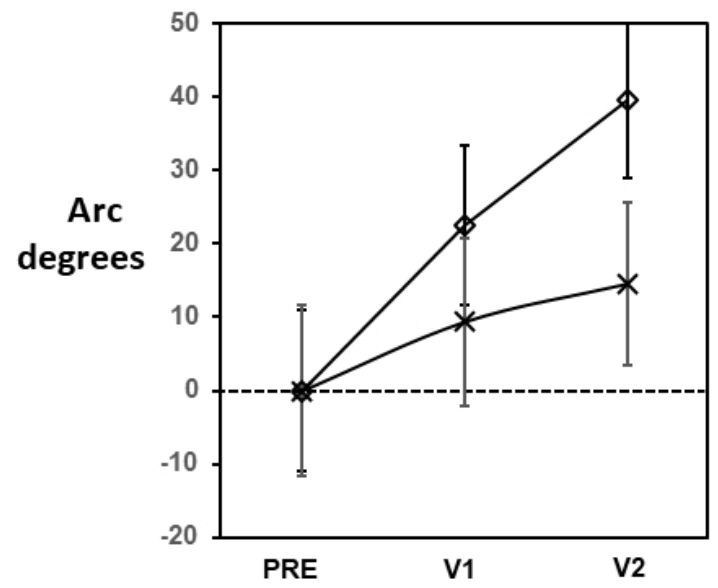

Figure 3. Rapid and marked improvement in active shoulder flexion by paretic arm after PSE treatment. Goniometry for mean changes in rotated angle (arc degrees) of A) active and B) passive shoulder flexion from baseline, comparing between the control $(x)$ and etanercept $(\diamond)$-treated groups $( \pm$ S.E). PRE: baseline; V1: Visit 1 after treatment 1; V2: Visit 2 after treatment. $* * \mathrm{p}<0.01$.

$$
190 \times 254 \mathrm{~mm}(96 \times 96 \mathrm{DPI})
$$


Figure 4

Figure 4. Change in pressure sensitivity by algometry. Comparison of etanercept versus control treatment groups for sensitivity to applied pressure by algometry on the A) paretic/hemiplegic arm versus B) unaffected arm. Mean change shown for the control $(x)$ and etanercept $(\diamond)$-treated groups $( \pm$ S.E). PRE: baseline; V1: Visit 1 after treatment 1; V2: Visit 2 after treatment. $\mathrm{p}>0.05$. Scale for applied pressure measured in Newtons (N).

$190 \times 254 \mathrm{~mm}(96 \times 96 \mathrm{DPI})$ 


\section{Figure 5}

Figure 5. Similar change in Fatigue Assessment Scores (FAS). Both control $(\times)$ and etanercept $(\diamond)$ groups $( \pm$ S.E) showed improvement in mean fatigue levels (lower values out of total 50) over the trial period. PRE: baseline; V2: Visit 2 after treatment 2; D30: day 30 after Visit 1. p > 0.05.

$190 \times 254 \mathrm{~mm}(96 \times 96 \mathrm{DPI})$ 
Figure 6

Figure 6. Quantitative thermosensory analysis. Change in recorded temperature (OC) for cold pain sensitivity of control $(x)$ versus etanercept $(\diamond)$ groups $( \pm$ S.E) during trial. PRE: baseline; V1: Visit 1 after treatment; V2: Visit 2 after treatment. $p>0.05$.

$190 \times 254 \mathrm{~mm}(96 \times 96 \mathrm{DPI})$ 